\title{
Bistructures, Bidomains and Linear Logic
}

Pierre-Louis Curien, LIENS, CNRS-Ecole Normale Supérieure, Paris, France Gordon Plotkin, LFCS, Comp. Sc. Dept., Edinburgh University, Scotland Glynn Winskel, BRICS ${ }^{1}$, Comp. Sc. Dept., Aarhus University, Denmark

\begin{abstract}
Bistructures are a generalisation of event structures which allow a representation of spaces of functions at higher types in an orderextensional setting. The partial order of causal dependency is replaced by two orders, one associated with input and the other with output in the behaviour of functions. Bistructures form a categorical model of Girard's classical linear logic in which the involution of linear logic is modelled, roughly speaking, by a reversal of the roles of input and output. The comonad of the model has an associated co-Kleisli category which is closely related to that of Berry's bidomains (both have equivalent non-trivial full sub-cartesian closed categories).
\end{abstract}

\section{Introduction}

In this paper we link Winskel's bistructures [25], Girard's linear logic [10] and Berry's bidomains [25]. We show how bistructures provide a model of classical linear logic extending Girard's web model $[10,11]$; we show too that a certain class of bistructures represent bidomains. We hope that the structures isolated here will help in the search for a direct, extensional and "mathematically natural" account of sequentiality and thereby of Milner's fully abstract model of PCF [20].

Girard has given an analysis of intuitionistic logic in terms of his more primitive linear logic. When we consider models, this is reflected in the fact that cartesian closed categories (categorical models of intuitionistic logic) arise as the co-Kleisli categories associated with categorical models of linear logic. In particular, linear logic yields refined analyses of the categories of domains used in denotational semantics. For instance, Berry and Curien's

\footnotetext{
${ }^{1}$ Basic Research in Computer Science, Centre of the Danish National Research Foundation.
} 
category of concrete data structures and sequential algorithms [5] may be obtained as the co-Kleisli category of a games model $[6,16]$. The connection between games and sequentiality has in turn informed recent work on intensional models of PCF and their fully-abstract extensional collapse $[1,12]$.

After Berry isolated the mathematically natural notion of stability [3], it was soon realized that sequential functions are stable. While there is a cartesian closed category of stable functions, at higher orders the extensional ordering is not respected. It was therefore natural for Berry to introduce bidomains. These are biorders - that is, sets equipped with two partial orders. One is an intensional stable ordering, based on the method of computation; the other is an extensional ordering, inherited from Scott's domain theory. Models of this kind can be viewed as mathematically tractable "approximations" to the desired sequential structures.

Event structures are partial orders of events equipped with a conflict relation and obeying an axiom of finite causes. They were introduced in [21] as a model of concurrency, and turned out to have close connections with concrete domains [14] and hence sequentiality [5]; they are also a natural generalisation of Girard's webs. Winskel introduced bistructures (of events) in [25], representing a full sub-cartesian closed category of bidomains. They are biorders equipped with a binary consistency relation; the two orders are obtained by decomposing the event structure order into left and right (input and output) components.

The main idea of this paper is that the inherent symmetry of bistructures enables one to obtain a model of classical linear logic, generalising the web model. The model is obtained by modifying the original definition-retaining its axiom of finite causes, but with all axioms symmetric. The configurations of a bistructure can be equipped with both a stable and an extensional ordering, that is they are biorders; further, the morphisms of the category of bistructures yield linear functions of the biorders (in a certain sense). Unfortunately not all biorders obtained in this way are bidomains; further not all linear functions come from morphisms of bistructures.

However by considering the co-Kleisli category and then restricting the allowed bistructures, one obtains a category equivalent to a full sub-cartesian closed category of Berry's category of bidomains and which provides a model of PCF. It has to be admitted that the situation here is not entirely as one would like: perhaps the notions of bistructures and bidomains should be adjusted. Ideal would be to have a bidomain model of classical linear logic, 
with a co-Kleisli category equivalent to that of stable continuous functions, and containing a (full) submodel equivalent to one of bistructures; further, there should be a representation theorem, that the bidomains corresponding to bistructures are precisely those satisfying suitable axioms.

It may be that a natural extensional account of sequentiality can be given within a "bistructural" framework. One can imagine replacing the stable ordering by a structure for sequentiality. If one does not know the right axioms, one could instead look for suitable variants of bistructures of events.

However, Loader's undecidability result [19] for the finitary fragment of PCF shows that there is a major obstacle to finding a category of structured sets providing a fully abstract model of PCF. We would expect that such structures would not be "finitary" in the sense that, say, partial orders and topological spaces are, but that measure spaces are not (note that the definition of measure spaces refers to the "external" notion of the natural numbers). ${ }^{2}$ It may nonetheless be possible to find suitable infinitary structure. The work in this paper suggests that one might do well to seek linear models whose co-Kleisli categories correspond to the sequential functions. There may even be enough symmetry that one has a model of classical linear logic.

In Sections 2 and 3 we give two approaches to bistructures; these represent two independent developments of the ideas of this paper [23, 7]. Section 2 starts from the world of webs and stable domain theory; Section 3 proceeds from that of event structures and continuous domain theory. We introduce bistructures in Section 4, and bistructure morphisms in Section 5. In Section 6 we show (Theorem 1) that bistructures provide a model of classical linear logic. In Section 7 we consider bidomains, establishing the connection with bistructures (Theorem 2). In Section 8 we discuss possible variations and connections with other work; in particular we consider strengthenings of bistructures incorporating Ehrhard's hypercoherences (see [8]) thereby accounting for strong stability within our approach.

In this paper, cpos are partial orders with a least element and lubs of all directed sets; continuous functions between cpos are those monotonic func-

\footnotetext{
${ }^{2}$ In fact, a notion of finitary category of structured sets can be formalised; one requires that the structures and morphisms are specified by formulas of higher-order logic referring only to the carrier sets. Then Loader's result implies that such a category cannot provide a fully abstract model of PCF, assuming that it is finitarily cartesian closed (in a suitable sense) and that the structured set corresponding to the Booleans has finite carrier.
} 
tions preserving all the directed lubs. For other domain-theoretic terminology see, for example, [28].

\section{Motivation from stability}

We recall the basics of Girard's stable model of classical linear logic [10, 11]. A web is a structure $(E, \smile)$, where:

- $E$ is a set of events (or tokens), and

- $\smile$ is a binary irreflexive symmetric relation of conflict (called strict incoherence in [10]).

Throughout this paper we use Girard's notation: $\asymp$ is the reflexive closure of the irreflexive relation $\smile$, and $\smile$, the complement of $\smile$, is the reflexive closure of the irreflexive relation $\frown$. It is clear that specifying one relation determines all the others.

The configurations (called cliques in [10]) of $(E, \smile)$ are the subsets $x \subseteq E$ which are

- consistent: $\forall e, e^{\prime} \in x \quad e \bigodot e^{\prime}$.

Ordered by inclusion, the configurations of $E$ form a cpo $(\Gamma(E), \subseteq)$; as a collection of sets, $\Gamma(E)$ is a coherence space in the sense of $[10,11]$. The webs form a category, taking the morphisms from $E_{0}$ to $E_{1}$ to be the stable functions from $\Gamma\left(E_{0}\right)$ to $\Gamma\left(E_{1}\right)$, i.e., those continuous functions $f$ such that whenever $e_{1} \in f(x)$ there is a minimum finite $x_{0} \subseteq x$ such that $e_{1} \in f\left(x_{0}\right)$. In this setting, the stable functions coincide with the conditionally multiplicative functions, i.e., the continuous functions that preserve binary compatible glbs (which are, categorically speaking, pullbacks).

The category is cartesian closed: the function space $E_{0} \rightarrow E_{1}$ has as events the pairs $\left(x, e_{1}\right)$ of a finite configuration of $E_{0}$ and an event of $E_{1}$, with incoherence defined by:

$$
\left(x, e_{1}\right) \asymp\left(y, e_{1}^{\prime}\right) \Leftrightarrow(x \uparrow y) \text { and }\left(e_{1} \asymp e_{1}^{\prime}\right)
$$

where $x \uparrow y$ means $\exists z \quad x, y \subseteq z$. The configurations of $E_{0} \rightarrow E_{1}$ are in 1-1 correspondence with the morphisms from $E_{0}$ to $E_{1}$, associating to each 
stable function $f$ its trace $\operatorname{tr}(f)$, consisting of those pairs $\left(x, e_{1}\right)$ such that $e_{1} \in f(x)$ and $e_{1} \notin f(y)$ if $y \subset x$. The inclusion of configurations determines an ordering on stable functions, refining the pointwise ordering and called the stable ordering [2].

The definition of $E_{0} \rightarrow E_{1}$ is asymmetric in that configurations are paired with events, rather than events with events. This led Girard to two successive decompositions, each of which turned out to have deep logical significance.

- First, $E_{0} \rightarrow E_{1}$ can be obtained as $\left(! E_{0}\right) \multimap E_{1}$, where, for any $E$, the web $E$ (the exponential of $E$, pronounced "bang $E$ ") has as events the finite configurations of $E$ (with $\subsetneq=\uparrow$ ), and where, for any $E_{0}, E_{1}$, the web $E_{0} \multimap E_{1}$, the linear function space, has as events pairs $\left(e_{0}, e_{1}\right)$ of events of $E_{0}$ and events of $E_{1}$, with incoherence defined by:

$$
\left(e_{0}, e_{1}\right) \asymp\left(e_{0}^{\prime}, e_{1}^{\prime}\right) \Leftrightarrow\left(e_{0} \frown e_{0}^{\prime}\right) \text { and }\left(e_{1} \asymp e_{1}^{\prime}\right)
$$

- Second, the remarkable symmetry between $\asymp$ and $\asymp$ in the definition of $E_{0} \multimap E_{1}$ leads to the decomposition $E_{0} \multimap E_{1}=\left(E_{0}^{\perp}\right) \wp E_{1}$, where, for any $E$, the web $E^{\perp}$, the linear negation of $E$, has the same events as $E$, but has as coherence the incoherence of $E$, and where, for any $E_{0}, E_{1}$, the web $E_{0} \wp E_{1}$ (the "par" of $E_{0}$ and $E_{1}$ ) has as events the pairs $\left(e_{0}, e_{1}\right)$ of an event of $E_{0}$ and an event of $E_{1}$, with incoherence defined by:

$$
\left(e_{0}, e_{1}\right) \asymp\left(e_{0}^{\prime}, e_{1}^{\prime}\right) \Leftrightarrow\left(e_{0} \asymp e_{0}^{\prime}\right) \text { and }\left(e_{1} \asymp e_{1}^{\prime}\right)
$$

Returning to the consideration of stable functions, let us see how to describe the pointwise order between stable functions at the level of traces. In $E_{0} \rightarrow E_{1}$ there arises a natural ordering between events $\left(x, e_{1}\right)$ if we vary only the input $x$ (whence the superscript ${ }^{L}$, for "left"):

$$
\left(x, e_{1}\right) \leq^{L}\left(y, e_{1}^{\prime}\right) \Leftrightarrow\left(y \subseteq x \text { and } e_{1}=e_{1}^{\prime}\right)
$$

Now define a partial order $\sqsubseteq$ on $\Gamma\left(E_{0} \rightarrow E_{1}\right)$ by:

$$
\phi \sqsubseteq \psi \Leftrightarrow \forall\left(x, e_{1}\right) \in \phi \exists y \subseteq x \quad\left(y, e_{1}\right) \in \psi
$$

or, equivalently:

$$
\phi \sqsubseteq \psi \Leftrightarrow \forall e \in \phi \exists e^{\prime} \in \psi e \leq^{L} e^{\prime}
$$


Then it is easy to see that for any two stable functions $f, g$ :

$$
(\forall x \quad f(x) \subseteq g(x)) \Leftrightarrow \operatorname{tr}(f) \sqsubseteq \operatorname{tr}(g)
$$

Since the stable ordering is a refinement of the pointwise ordering, it makes sense to ask whether there exists a sensible "complement" of the stable ordering. Indeed we shall see in Proposition 1 that we can always factor $\phi \sqsubseteq \psi$ uniquely as $\phi \sqsubseteq^{L} \chi \subseteq \psi$. Here $\phi \sqsubseteq^{L} \chi$ means that $\phi \sqsubseteq \chi$ and $\chi$ is minimal with respect to inclusion (i.e., the stable ordering) among all $\chi^{\prime}$ such that $\phi \sqsubseteq \chi^{\prime}$; in other words, $\chi$ is "the part of $\psi$ showing that $\phi \sqsubseteq \psi$ " (notice that, given $\left(x, e_{1}\right)$, the $y$ in the definition of $\phi \sqsubseteq \psi$ is unique).

So far, our discussion has been implicitly carried at first-order types, where we have stable functions that can be ordered in two ways ( $\subseteq$ and $\sqsubseteq$ ). If we next consider second-order types, or functionals, the explicit consideration of both the pointwise and the stable orderings at first-order types leads us to focus on functionals that are not only stable with respect to the stable ordering, but also monotonic with respect to the pointwise ordering. That is, we want to retain only those stable functionals $H$ from $\Gamma\left(E_{0} \rightarrow E_{1}\right)$ to $\Gamma\left(E_{2}\right)$ such that:

$$
\forall \phi, \psi(\phi \sqsubseteq \psi \Rightarrow H(\phi) \subseteq H(\psi))
$$

(where we now freely confuse functions with their traces), which, by the $\subseteq$-monotonicity of $H$ and the definition of $\complement^{L}$, can be rephrased as:

$$
\forall \phi, \psi\left(\phi \sqsubseteq^{L} \psi \Rightarrow H(\phi) \subseteq H(\psi)\right)
$$

Now, specialising to finite $\phi$ and $\psi$, suppose that $\left(\phi, e_{2}\right) \in H$. Then we must have that $e_{2} \in H(\psi)$, i.e., there must exist $\left(\psi_{0}, e_{2}\right)$ in $H$ such that $\psi_{0} \subseteq \psi$. Therefore we ask for the following condition, called the securedness condition:

$$
\forall e \in H \quad \forall e^{\prime} \quad\left(e^{\prime} \leq^{R} e \Rightarrow \exists e^{\prime \prime} \in H \quad e^{\prime} \leq^{L} e^{\prime \prime}\right)
$$

where the order $\leq^{R}$ is defined by

$$
\left(\psi, e_{2}^{\prime}\right) \leq^{R}\left(\phi, e_{2}\right) \Leftrightarrow\left(\phi \sqsubseteq^{L} \psi \text { and } e_{2}^{\prime}=e_{2}\right)
$$

To summarise, by going from base types successively to first-order and then to second-order types, we have identified two orderings on events. 
- The $\leq^{L}$ ordering allows us to describe the extensional ordering between traces.

- The securedness condition, which involves both orderings $\leq^{L}$ and $\leq^{R}$, allows us to capture the preservation of this extensional ordering by functionals.

This suggests that we consider structures $\left(E, \leq^{L}, \leq^{R}, \smile\right)$, where $(E, \smile)$ is a web, with the aim of building a cartesian closed category of biordered domains (cf. the introduction), and, as it turns out, a model of classical linear logic.

\section{Motivation from continuity}

In event structures (which predate Girard's webs), a causal dependency relation inspired from Petri net theory is considered in addition to the conflict relation [21]. In full, an event structure is a structure $(E, \leq, \smile)$ where $^{3}$ :

- $E$ is a set of events,

- $\leq$ is a partial order of causal dependency, and

- $\smile$ is a binary, irreflexive, symmetric relation of conflict.

The configurations (or states) of such an event structure are those subsets $x \subseteq E$ which are:

- consistent: $\forall e, e^{\prime} \in x e \leftrightharpoons e^{\prime}$, and

- left closed: $\forall e, e^{\prime} \in E \quad e^{\prime} \leq e \in x \Rightarrow e^{\prime} \in x$.

Ordered by inclusion, the configurations form a coherent prime algebraic domain $(\Gamma(E), \subseteq)[21]$; such domains are precisely the infinitely distributive, coherent Scott domains [27]. An instance of the causal dependency ordering $e^{\prime} \leq e$ when $e$ and $e^{\prime}$ are distinct, is understood as meaning that the event $e$ causally depends on the event $e^{\prime}$, in that the event $e$ can only occur after $e^{\prime}$ has occurred. Given this understanding it is reasonable to impose a finiteness axiom, expressing that an event has finite causes:

\footnotetext{
${ }^{3}$ In [21], an axiom relating causal dependency and conflict is imposed; however it is inessential in that it does not affect the class of domains represented.
} 


$$
\left\{e^{\prime} \mid e^{\prime} \leq e\right\} \text { is finite, for all events } e \text {. }
$$

The event structures satisfying this axiom yield the dI-domains [2] which are coherent, and therefore lead to a cartesian closed category of stably ordered stable functions. (See [26] where an alternative description of event structures using an enabling relation instead of an ordering on events is used to give a simple description of the function space construction.)

But event structures can also be used to describe a continuous model of intuitionistic linear logic, equivalent to the category of coherent prime algebraic domains, with completely additive functions (i.e., functions preserving arbitrary lubs - just called "additive" below). We take as objects event structures (but without the axiom of finite causes: this is the price to pay), and as morphisms configurations of a "function space" of event structures. Let $E_{i}=\left(E_{i}, \leq_{i}, \smile_{i}\right), i=0,1$, be event structures. Define:

$$
\begin{aligned}
& E_{0} \multimap E_{1}=\left(E_{0} \times E_{1}, \leq, \smile\right) \\
\text { where } & \left(e_{0}, e_{1}\right) \leq\left(e_{0}^{\prime}, e_{1}^{\prime}\right) \Leftrightarrow e_{0}^{\prime} \leq_{0} e_{0} \text { and } e_{1} \leq_{1} e_{1}^{\prime}, \\
\text { and } & \left(e_{0}, e_{1}\right) \smile\left(e_{0}^{\prime}, e_{1}^{\prime}\right) \Leftrightarrow e_{0} \bigodot_{0} e_{0}^{\prime} \text { and } e_{1} \smile_{1} e_{1}^{\prime} .
\end{aligned}
$$

The configurations of $E_{0} \multimap E_{1}$ are in 1-1 correspondence with the additive functions from $\Gamma\left(E_{0}\right)$ to $\Gamma\left(E_{1}\right)$-additive functions are determined by their action on complete primes ${ }^{4}$ which correspond to events. The configuration associated with an additive function $f$ is its graph, consisting of those pairs $\left(e_{0}, e_{1}\right)$ such that $e_{1} \in f\left(\left\{e_{0}^{\prime} \mid e_{0}^{\prime} \leq e_{0}\right\}\right)$.

The inclusion ordering on configurations reflects the pointwise ordering on functions; in particular, the function events $\left(e_{0}, e_{1}\right)$ correspond to the prime additive one-step functions (see [31]); and the order $\leq$ to the pointwise order between them.

A morphism $E_{0} \rightarrow E_{1}$ is defined to be a configuration of $E_{0} \multimap E_{1}$. As such it is a relation between the events of $E_{0}$ and $E_{1}$. Composition in the category is that of relations. The category is a model of intuitionistic linear logic, as defined in $[24,4]$. For instance, its tensor is given in a coordinatewise

\footnotetext{
${ }^{4} \mathrm{~A}$ complete prime of a Scott domain $(D, \sqsubseteq)$ is an element $p$ for which whenever $X$ is bounded above and $p \sqsubseteq \bigsqcup X$ then $p \sqsubseteq x$ for some $x$ in $X$. Complete primes are $a$ fortiori compact, where the definition of compact is obtained by replacing " $X$ is bounded above" by " $X$ is directed".
} 
fashion. For event structures $E_{i}=\left(E_{i}, \leq_{i}, \smile_{i}\right)$, for $i=0,1$, define:

$$
\begin{aligned}
& E_{0} \otimes E_{1}=\left(E_{0} \times E_{1}, \leq, \smile\right) \\
\text { where } & \left(e_{0}, e_{1}\right) \leq\left(e_{0}^{\prime}, e_{1}^{\prime}\right) \Leftrightarrow e_{0} \leq_{0} e_{0}^{\prime} \text { and } e_{1} \leq_{1} e_{1}^{\prime}, \\
\text { and } \quad & \left(e_{0}, e_{1}\right) \subseteq\left(e_{0}^{\prime}, e_{1}^{\prime}\right) \Leftrightarrow e_{0} \bigodot_{0} e_{0}^{\prime} \text { and } e_{1} \bigodot_{1} e_{1}^{\prime} .
\end{aligned}
$$

Monoidal-closure follows from the isomorphism

$$
\left(E_{0} \otimes E_{1} \multimap E_{2}\right) \cong\left(E_{0} \multimap\left(E_{1} \multimap E_{2}\right)\right)
$$

natural in $E_{0}$ and $E_{2}$. Product and coproduct are obtained by disjoint juxtaposition of event structures, extending conflict across the two event sets in the case of coproduct. The comonad operation is:

$$
! E=\left(\Gamma(E)^{0}, \subseteq, \smile\right)
$$

for an event structure $E$, with events the compact configurations $\Gamma(E)^{0}$, and where $\smile$ stands for incompatibility with respect to inclusion. The continuous functions $\Gamma\left(E_{0}\right) \rightarrow \Gamma\left(E_{1}\right)$, between configurations of event structures $E_{0}, E_{1}$, are in 1-1 correspondence with the configurations of $! E_{0} \multimap E_{1}$.

Notice that this does not yield a model of classical linear logic. The reader should compare the asymmetric definition of conflict in $E_{0} \multimap E_{1}$ given above to capture continuity with the symmetric definition of incoherence in the stable framework (cf. Section 2).

Moreover, in this model of intuitionistic linear logic, all hope of considering the order $\leq$ as causal dependency is lost. The difficulty stems from the definition of the order $\leq$ for $\left(E_{0} \multimap E_{1}\right)$. Its events are ordered by:

$$
\left(e_{0}, e_{1}\right) \leq\left(e_{0}^{\prime}, e_{1}^{\prime}\right) \Leftrightarrow e_{0}^{\prime} \leq_{0} e_{0} \text { and } e_{1} \leq_{1} e_{1}^{\prime}
$$

The reversal in the $\leq_{0}$ order can lead to $\leq$ violating the axiom of finite causes, even though $\leq_{0}$ and $\leq_{1}$ do not: an infinite, ascending chain of events in $E_{0}$ can give rise to an infinite, descending chain in $E_{0} \multimap E_{1}$. Of course, there is no reason why the extensional ordering on functions should be a relation of causal dependency, so it was not to be expected that its restriction to step functions should be finitary.

However, if we factor $\leq$ into two orderings, one associated with input (on the left) and one with output (on the right), we can expose two finitary 
orderings. Define

$$
\begin{aligned}
& \left(e_{0}, e_{1}\right) \leq^{L}\left(e_{0}^{\prime}, e_{1}^{\prime}\right) \Leftrightarrow e_{0}^{\prime} \leq_{0} e_{0} \text { and } e_{1}=e_{1}^{\prime}, \\
& \left(e_{0}, e_{1}\right) \leq^{R}\left(e_{0}^{\prime}, e_{1}^{\prime}\right) \Leftrightarrow e_{0}^{\prime}=e_{0} \text { and } e_{1} \leq_{1} e_{1}^{\prime} .
\end{aligned}
$$

Then, it is clear that $\leq$ factors as

$$
\left(e_{0}, e_{1}\right) \leq\left(e_{0}^{\prime}, e_{1}^{\prime}\right) \Leftrightarrow\left(e_{0}, e_{1}\right) \leq^{L}\left(e_{0}^{\prime}, e_{1}\right) \text { and }\left(e_{0}^{\prime}, e_{1}\right) \leq^{R}\left(e_{0}^{\prime}, e_{1}^{\prime}\right),
$$

and that this factorisation is unique. Provided the orderings of $E_{0}$ and $E_{1}$ are finitary, then so are $\leq^{R}$ and $\geq^{L}$. This factorisation is the first step towards the definition of bistructures. To indicate its potential, and to further motivate bistructures, we study a simple example.

Let $E_{0}$ and $E_{1}$ be the event structures shown below. Both have empty conflict relations. Taking advantage of the factorisation we have drawn them alongside the additive function space $E_{0} \multimap E_{1}$.

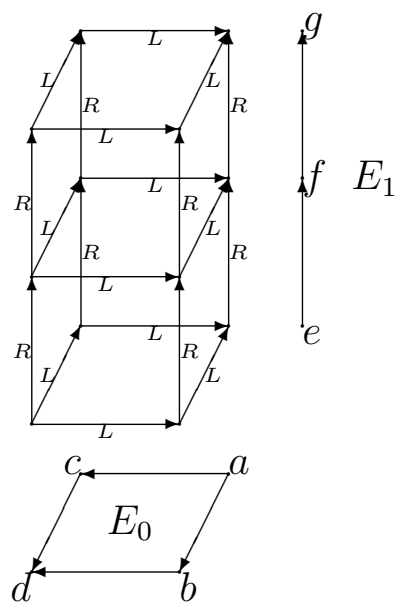

The conflict relation of $E_{0} \multimap E_{1}$ is empty. So here an additive function from $\Gamma\left(E_{0}\right)$ to $\Gamma\left(E_{1}\right)$ is represented by a $\leq$-downwards-closed subset of events of $E_{0} \multimap E_{1}$. For instance, the events in the diagram (below left) are associated with the function that outputs $e$ on getting input event $a$, outputs $f$ for input $b$ or $c$, and outputs $g$ for input $d$. The extensional ordering on functions corresponds to inclusion on $\leq$-downwards-closed subsets of events. It is clear that such a function is determined by specifying the minimal input events which yield some specific output (shown in the diagram below right). This amounts to the subset of $\leq^{L}$-maximal events of the function, and we can 
call this subset the trace of the function. Notice, though, that this particular function is not stable; output $f$ can be obtained for two non-conflicting but distinct events $b$ and $c$. A stable function should not have $\leq^{L}$-downwards compatible distinct events in its trace.
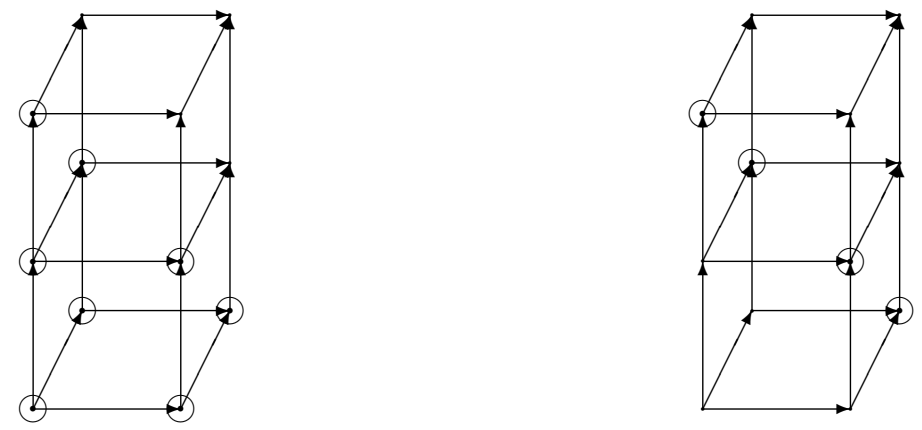

For stable functions, the stable ordering is obtained as inclusion of traces. For example:

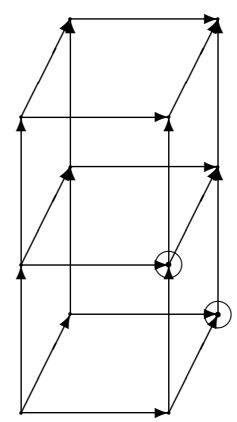

is stable below

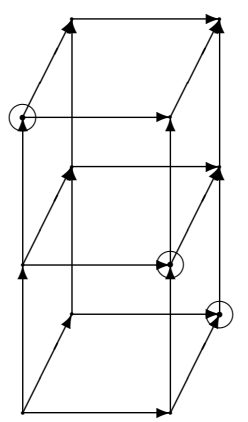

Notice that traces $\phi$ of additive functions from from $\Gamma\left(E_{0}\right)$ to $\Gamma\left(E_{1}\right)$ are secured, in the sense that:

$$
\left(e \in \phi \text { and } e^{\prime} \leq^{R} e\right) \Rightarrow\left(\exists e^{\prime \prime} \in \phi e^{\prime} \leq^{L} e^{\prime \prime}\right)
$$

or more concretely:

$$
\left(\left(e_{0}, e_{1}\right) \in \phi \text { and } e_{1}^{\prime} \leq e_{1}\right) \Rightarrow\left(\exists e_{0}^{\prime}\left(e_{0}^{\prime}, e_{1}^{\prime}\right) \in \phi \text { and } e_{0}^{\prime} \leq e_{0}\right)
$$

This is the same securedness condition that appeared in Section 2. Here we can understand the condition as saying that for any output, lesser output must arise through the same or lesser input.

Let us summarise this discussion. 
- The graphs of additive functions are the $\leq$-downwards-closed, consistent subsets of events.

- The extensional order corresponds to inclusion of graphs.

- The traces of functions are the sets of $\leq^{L}$-maximal events of their graphs.

- The stable order corresponds to inclusion of traces.

These observations, based on the continuous model construction, will, as it turns out, also make sense in a biordered framework. They encourage us to consider bistructures $\left(E, \leq^{L}, \leq^{R}, \smile\right)$ and provide guidance as to which axioms we should impose on $\leq^{L}, \leq^{R}$, and $\subsetneq$. One expects a function-space construction that maintains both stable and extensional orderings, corresponding to taking as morphisms those functions which are continuous with respect to the extensional ordering and stable with respect to the stable ordering.

We end the section with a remark. One might wonder why we have explicitly considered an ordering $\leq$ on events to describe a cartesian closed category of continuous functions, while webs suffice for the purpose of building a cartesian closed category of stable functions. The reason is that the treatment of stability is based on traces of functions, while the treatment of continuity is based on their graphs. Graphs of continuous functions ${ }^{5}$ are upwards closed in their first component, even if the underlying event structure has a trivial partial order, and we need an order relation on events to capture that fact.

\section{Bistructures}

The following definition of bistructures allows us to fulfill the hopes expressed in the previous sections.

Definition $1 A$ (countable) bistructure is a structure $\left(E, \leq^{L}, \leq^{R}, \frown\right)$ where $E$ is a countable set of events, $\leq^{L}, \leq^{R}$ are partial orders on $E$ and $\frown$ is a binary reflexive, symmetric relation on $E$ such that:

\footnotetext{
${ }^{5}$ The graph of a continuous function $f$ from $\Gamma\left(E_{0}\right)$ to $\Gamma\left(E_{1}\right)$ consists of all pairs $\left(x, e_{1}\right)$ with $x$ compact such that $e_{1} \in f(x)$.
} 
1. defining $\leq=\left(\leq^{L} \cup \leq^{R}\right)^{\star}$, we have the following factorisation property:

$$
e \leq e^{\prime} \Rightarrow \exists e^{\prime \prime} e \leq^{L} e^{\prime \prime} \leq^{R} e^{\prime}
$$

2. defining $\preceq=\left(\geq^{L} \cup \leq^{R}\right)^{\star}$,

(a) $\preceq$ is finitary, i.e., $\left\{e^{\prime} \mid e^{\prime} \preceq e\right\}$ is finite, for all $e$,

(b) $\preceq$ is a partial order;

3. (a) $\downarrow^{L} \subseteq \asymp \quad$ (b) $\uparrow^{R} \subseteq \bigodot$

The two compatibility relations are defined by:

$$
\begin{aligned}
& e \downarrow^{L} e^{\prime} \Leftrightarrow \exists e^{\prime \prime} e^{\prime \prime} \leq^{L} e \text { and } e^{\prime \prime} \leq^{L} e^{\prime}, \\
& e \uparrow^{R} e^{\prime} \Leftrightarrow \exists e^{\prime \prime} e \leq^{R} e^{\prime \prime} \text { and } e^{\prime} \leq^{R} e^{\prime \prime} .
\end{aligned}
$$

Notice the symmetry of the axioms. They are invariant under the "duality":

$$
\begin{aligned}
& \leq^{L} \mapsto \geq^{R}, \\
& \leq^{R} \mapsto \geq^{L}, \\
& \frown \mapsto
\end{aligned}
$$

which is why we obtain a model of the classical logic. Bistructures of the form $\left(E, i d_{E}, \leq, \smile\right)$, i.e., such that the $\leq^{L}$ order is degenerate, are essentially the ordinary, countable event structures, $(E, \leq, \smile)$, satisfying the axiom of finite causes. We say "essentially" because Axiom 3(b) is not part of the above definition of event structure, but does not restrict the class of domains represented.

Remark 1 In the presence of Axiom 2(a), Axiom 2(b) is equivalent to requiring that $e \prec e^{\prime}$ is well-founded, where $e \prec e^{\prime}$ means $e \preceq e^{\prime}$ and $e \neq e^{\prime}$. (A fortiori, $<^{R}$ and $>^{L}$ are well-founded.)

The axioms of bistructures are strong enough to imply the uniqueness of the decomposition of $\leq=\left(\leq^{L} \cup \leq^{R}\right)^{\star}$, and also that $\leq$ is a partial order.

Lemma 1 Let $E$ be a bistructure. The following properties hold, for all e, $e^{\prime}$ in $E$ :

$$
\begin{aligned}
& \text { (1) }\left(e \downarrow^{L} e^{\prime} \text { and } e \subsetneq e^{\prime}\right) \Rightarrow e=e^{\prime} \text {, } \\
& \text { (2) }\left(e \downarrow^{L} e^{\prime} \text { and } e \uparrow^{R} e^{\prime}\right) \Rightarrow e=e^{\prime} \text {, } \\
& \text { (3) } e \leq e^{\prime} \quad \Rightarrow \exists ! e^{\prime \prime} e \leq^{L} e^{\prime \prime} \leq^{R} e^{\prime}
\end{aligned}
$$


Proof. (1) If $e \downarrow^{L} e^{\prime}$, then $e \asymp e^{\prime}$, which together with $e \frown e^{\prime}$ implies $e=e^{\prime}$ by definition of $\asymp$.

(2) Immediate consequence of (1) since $e \uparrow^{R} e^{\prime}$ implies $e \subsetneq e^{\prime}$.

(3) Suppose that $e \leq^{L} e^{\prime \prime} \leq^{R} e^{\prime}$ and $e \leq^{L} e^{\prime \prime \prime} \leq^{R} e^{\prime}$. Then $e^{\prime \prime} \downarrow^{L} e^{\prime \prime \prime}$ and $e^{\prime \prime} \uparrow^{R} e^{\prime \prime \prime}$, therefore $e^{\prime \prime}=e^{\prime \prime \prime}$ by $(1)$.

The unique factorisation property enables a diagrammatic style of proof.

Lemma 2 The relation $\leq$ defined in Axiom 1 of bistructures is a partial order.

Proof. The relation $\leq$ is certainly reflexive and transitive. With the aim of proving antisymmetry, suppose $e \leq e^{\prime}$ and $e^{\prime} \leq e$. Then pictorially by factorising $\leq$, for some events $\varepsilon$ and $\varepsilon^{\prime}$, we have:

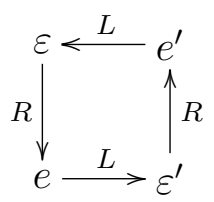

So $e \leq \varepsilon$, and factorising this we get $e \leq^{L} \varepsilon^{\prime \prime} \leq^{R} \varepsilon \leq^{R} e$, for some $\varepsilon^{\prime \prime}$. But $e \leq^{L} e \leq^{R} e$ so the uniqueness of factorisation gives $e=\varepsilon^{\prime \prime}$. Then as $\leq^{R}$ is a partial order $e=\varepsilon$. Therefore the above picture collapses to:

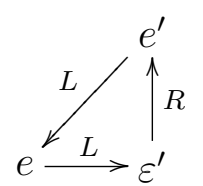

The uniqueness of the factorisation of $e^{\prime} \leq e^{\prime}$ gives $\varepsilon^{\prime}=e^{\prime}$, so as $\leq^{L}$ is a partial order, $e=e^{\prime}$, as required.

As with Girard's webs, bistructures provide a concrete level of description of abstract points, or configurations, which we define next.

Definition $2 A$ configuration of a bistructure $\left(E, \leq^{L}, \leq^{R}, \bigodot\right)$ is a subset $x \subseteq E$ which is:

- consistent: $\forall e, e^{\prime} \in x \quad e \smile e^{\prime}$, and 
- secured: $\forall e \in x \forall e^{\prime} \leq^{R} e \exists e^{\prime \prime} \in x e^{\prime} \leq^{L} e^{\prime \prime}$.

[Notice that $e^{\prime \prime}$ is unique in any consistent set because of Axiom 3(a) on bistructures.] Write $\Gamma(E)$ for the set of configurations of a bistructure $E$, and $\Gamma(E)^{0}$ for the set of finite configurations (see Proposition 2).

When $\leq^{L}=i d$, the securedness condition amounts to $\leq^{R}$-downwardsclosure, hence in that case configurations are just the configurations of the underlying event structure $\left(E, \leq^{R}, \smile\right)$.

We next define order relations on configurations.

Definition 3 Let $E$ be a bistructure. We define the stable ordering $5^{R}$ and the extensional ordering $\sqsubseteq$ on configurations by:

$$
\begin{aligned}
& \sqsubseteq^{R} \text { is set-theoretic inclusion, } \\
& x \sqsubseteq y \Leftrightarrow \forall e \in x \quad \exists e^{\prime} \in y \quad e \leq^{L} e^{\prime}
\end{aligned}
$$

It follows from these definitions and from the reflexivity of $\leq^{L}$ that $\bigsqcup^{R}$ is included in $\sqsubseteq$. We define a third relation $\sqsubseteq^{L}$ as follows:

$$
x \sqsubseteq^{L} y \Leftrightarrow x \sqsubseteq y \text { and }\left(\forall z \in \Gamma(E) \quad\left(x \sqsubseteq z \quad \text { and } z \sqsubseteq^{R} y\right) \Rightarrow y=z\right)
$$

Thus, $x \sqsubseteq^{L} y$ means that $y$ is a $\sqsubseteq^{R}$-minimal configuration such that $x \sqsubseteq y$. Write $x \uparrow^{R} y$ for $\left(\exists z \in \Gamma(E) x, y \sqsubseteq^{R} z\right)$.

Some elementary properties of configurations are given in the following lemmas.

Lemma 3 Let $E$ be a bistructure, and suppose that $x \in \Gamma(E)$. If $e$ is in the $\leq$-downwards-closure of $x$, then it is in the $\leq^{L}$-downwards-closure of $x$.

Proof. Let $e^{\prime}$ in $x$ be such that $e \leq e^{\prime}$ :

$$
\begin{aligned}
& \exists e^{\prime \prime} e \leq^{L} e^{\prime \prime} \leq^{R} e^{\prime} \quad \text { by factorisation, } \\
& \exists e^{\prime \prime \prime} \in x \quad e^{\prime \prime} \leq^{L} e^{\prime \prime \prime} \quad \text { by securedness }
\end{aligned}
$$

Then $e \leq^{L} e^{\prime \prime \prime}$, which completes the proof.

It follows from Lemma 3 that $x \sqsubseteq y$ is equivalently defined by stating that the $\leq$-downwards-closure of $x$ is included in the $\leq$-downwards-closure of $y$. This characterisation is in accordance with the discussion in Section 3: compare $\sqsubseteq^{R}$ and $\sqsubseteq$ with graph and trace inclusion, respectively. 
Lemma 4 Let $E$ be a bistructure, and suppose that $x, y$ are in $\Gamma(E)$. If $x \uparrow^{R} y, e \in x, e^{\prime} \in y$, and $e \downarrow^{L} e^{\prime}$, then $e=e^{\prime}$.

Proof. Let $z \in \Gamma(E)$ be such that $x \sqsubseteq^{R} z$ and $y \sqsubseteq^{R} z$. We have $e \in z$ and $e^{\prime} \in z$, hence $e \smile e^{\prime}$. On the other hand, by Axiom 3(a) we find that $e \asymp e^{\prime}$, hence $e=e^{\prime}$.

Remark 2 Lemma 4 has two interesting consequences.

- If $x$ is a configuration and $e \in x$, then $e$ is $\leq^{L}$-maximal in $x$. In turn, this implies the antisymmetry of $\sqsubseteq$, which is thus a partial order (reflexivity and transitivity are immediate). In turn, the antisymmetry of $\sqsubseteq$ entails the reflexivity of $\sqsubseteq^{L}$.

- If $x \uparrow^{R} y$, then the set intersection $x \cap y$ is the glb of $x$ and $y$ with respect to both $\sqsubseteq^{R}$ and $\sqsubseteq$.

Definition 4 For $x$ in $\Gamma(E)$, we define the relativised relation $\preceq_{x}$ as the reflexive, transitive closure of $\preceq_{x}^{1}$ where:

$$
e \preceq_{x}^{1} e^{\prime} \Leftrightarrow{ }_{\text {def }} e \in x \text { and } e^{\prime} \in x \text { and } \exists e^{\prime \prime} e \geq^{L} e^{\prime \prime} \leq^{R} e^{\prime} .
$$

Lemma 5 Let $E$ be a bistructure. The following property holds, for all $x, y$ in $\Gamma(E)$ and $e$ in $E$ :

$$
\left(x \uparrow^{R} y \text { and } e \in x \cap y\right) \Rightarrow\left(\forall e^{\prime} \in E \quad e^{\prime} \preceq_{x} e \Leftrightarrow e^{\prime} \preceq_{y} e\right)
$$

(or, equivalently, in asymmetric form: $\left(x \uparrow^{R} y, e \in x \cap y\right.$ and $\left.e^{\prime} \preceq_{x} e\right) \Rightarrow$ $\left.e^{\prime} \in y\right)$.

Proof. It is clearly enough to show this for the one step relations $\preceq_{x}^{1}$ and $\preceq_{y}^{1}$. Suppose $e^{\prime} \preceq_{x}^{1} e$, and let $e^{\prime \prime}$ be such that $e^{\prime} \geq^{L} e^{\prime \prime} \leq^{R} e$. Since $y$ is secured, and since $e \in y$, there exists $e^{\prime \prime \prime}$ in $y$ such that $e^{\prime \prime} \leq^{L} e^{\prime \prime \prime}$. By Lemma 4 applied to $x, y, e^{\prime}$, and $e^{\prime \prime \prime}$, we get $e^{\prime}=e^{\prime \prime \prime} \in y$.

In the rest of this section, we examine some of the properties of the relations $\sqsubseteq^{R}$, the ground for the definition of exponentials in Section 6, while Propositions 2 and 3 concern completeness properties. 
Definition 5 For $x$ in $\Gamma(E)$ and a subset $z \subseteq x$, define

$$
[z]_{x}=\left\{e_{0} \in x \quad \mid \exists e \in z \quad e_{0} \preceq_{x} e\right\}
$$

If $z$ is a singleton $\{e\}$, we write simply $[e]_{x}$.

Lemma 6 Let $E$ be a bistructure, and suppose that $x \in \Gamma(E)$ and $z \subseteq x$. Then $[z]_{x}$ is a configuration which is finite if $z$ is finite and such that:

$$
z \subseteq[z]_{x} \sqsubseteq^{R} x \quad \text { and } \quad\left(\forall y \in \Gamma(E) \quad\left(z \subseteq y \uparrow^{R} x\right) \Rightarrow\left([z]_{x} \sqsubseteq^{R} y\right)\right)
$$

Proof. We show that $[z]_{x}$ is a configuration. It is clearly consistent, as it is a subset of $x$. If $e \in[z]_{x}$ and $e_{1} \leq^{R} e$, since $x$ is secured, there exists $e_{2}$ in $x$ such that $e_{1} \leq^{L} e_{2}$, and then $e_{2} \in[z]_{x}$ by the construction of $[z]_{x}$. Thus $[z]_{x}$ is a configuration. The finiteness property follows from Axiom 2(a). The rest of the statement is an immediate consequence of Lemma 5.

Remark 3 For any e the following "canonical" set

$$
[e]=\left\{e^{\prime} \mid e^{\prime} \leq^{R} e\right\}
$$

is a configuration containing $e$, and if $x$ is any other such, then $[e] \sqsubseteq x$. In contrast, there need be no $\subseteq$-least configuration containing a given e (cf. Lemma 6).

Proposition 1 Let $\sqsubseteq^{R}$, , and $\sqsubseteq^{L}$ be the relations on configurations defined above. The following properties hold:

1. $\sqsubseteq i s\left(\sqsubseteq^{L} \cup \sqsubseteq^{R}\right)^{\star}$, and satisfies Axiom 1,

2. for all configurations $x, y$ :

$$
x \sqsubseteq^{L} y \Leftrightarrow\left(x \sqsubseteq y \text { and } \forall e \in y \exists e_{0} \in x, e_{1} \in y \quad e \preceq_{y} e_{1} \geq^{L} e_{0}\right)
$$

3. $\sqsubseteq^{L}$ is a partial order, and

4. for all configurations $x, y$ with $x \sqsubseteq y$ there is a unique $z$ such that $x \sqsubseteq^{L} z \sqsubseteq^{R} y$. 
Proof. (1) Suppose $x \sqsubseteq y$. The subset $z=\left\{e_{1} \in y \mid \exists e_{0} \in x \quad e_{0} \leq^{L} e_{1}\right\}$ represents the part of $y$ actually used to check $x \sqsubseteq y$. But we have to close this subset to make it secured. Thus we consider $z_{1}=[z]_{y}$, which is a configuration by Lemma 6 . We show $x \sqsubseteq^{L} z_{1}$. Suppose that $x \sqsubseteq z_{1}^{\prime} \sqsubseteq^{R} z_{1}$, and let $e$ be an element of $z_{1}$. By the construction of $z_{1}$, there are $e_{0}$ in $x$ and $e_{1}$ in $y$ such that $e \preceq_{y} e_{1} \geq^{L} e_{0}$. Since $x \sqsubseteq z_{1}^{\prime}, e_{0} \leq^{L} e_{1}^{\prime}$ for some $e_{1}^{\prime}$ in $z_{1}^{\prime}$. Applying Lemma 4 to $y, z_{1}^{\prime}, e_{1}$, and $e_{1}^{\prime}$, we get $e_{1}=e_{1}^{\prime}$, hence $e_{1} \in z_{1}^{\prime}$, which implies $e \in z_{1}^{\prime}$ by Lemma 5. Therefore $z_{1} \sqsubseteq^{R} z_{1}^{\prime}$, which completes the proof that $x \sqsubseteq^{L} z_{1}$. The decomposition $x \sqsubseteq^{L} z_{1} \sqsubseteq^{R} y$ shows that $\sqsubseteq$ is contained in $\left(\complement^{L} \cup \bigsqcup^{R}\right)^{\star}$. The converse inclusion is obvious.

(2) follows immediately from the proof of (1), observing that, in full:

$$
z_{1}=\left\{e \in y \quad \mid \exists e_{0} \in x, e_{1} \in y \quad e \preceq_{y} e_{1} \geq^{L} e_{0}\right\}
$$

(3) The antisymmetry follows from the inclusion of $\complement^{L}$ in $\sqsubseteq$. The reflexivity of $\sqsubseteq^{L}$ has been already pointed out in remark 2. Suppose that $x \sqsubseteq^{L} y^{\prime} \sqsubseteq^{L} y$. Clearly $x \sqsubseteq y$, so with an eye to using (2) to show $x \sqsubseteq^{L} y$ suppose $e \in y$. By (2), there exist $e_{0}$ in $y^{\prime}, e_{1}$ in $y, e_{0}^{\prime}$ in $x$ and $e_{1}^{\prime}$ in $y^{\prime}$ such that

$$
\begin{aligned}
& e_{0} \leq^{L} e_{1} \text { and } e \preceq_{y} e_{1}, \\
& e_{0}^{\prime} \leq^{L} e_{1}^{\prime} \text { and } e_{0} \preceq_{y^{\prime}} e_{1}^{\prime} .
\end{aligned}
$$

Or in full:

$$
\begin{array}{ll}
e_{0} \leq^{L} e_{1} \geq^{R} e_{2} \cdots \leq^{L} e_{2 i+1}=e & \text { with } e_{2 j+1} \in y \text { for all } 0 \leq j \leq i, \\
e_{0}^{\prime} \leq^{L} e_{1}^{\prime} \geq^{R} e_{2}^{\prime} \cdots \leq^{L} e_{2 i^{\prime}+1}^{\prime}=e_{0} & \text { with } e_{2 j+1}^{\prime} \in y^{\prime} \text { for all } 0 \leq j \leq i^{\prime}
\end{array}
$$

Since $y^{\prime} \sqsubseteq y$ and $e_{1}^{\prime} \in y^{\prime}$, there exists $e_{1}^{\prime \prime}$ such that $e_{1}^{\prime} \leq^{L} e_{1}^{\prime \prime}$ and $e_{1}^{\prime \prime} \in y$. Since $e_{2}^{\prime} \leq^{R} e_{1}^{\prime} \leq^{L} e_{1}^{\prime \prime}$, there exists $e_{2}^{\prime \prime}$ such that $e_{2}^{\prime} \leq^{L} e_{2}^{\prime \prime} \leq^{R} e_{1}^{\prime \prime}$, by factorisation. Since $y$ is secured, there exists $e_{3}^{\prime \prime}$ in $y$ such that $e_{2}^{\prime \prime} \leq^{L} e_{3}^{\prime \prime}$. In order to continue this lifting of the $e_{i}^{\prime}$ relative to $y^{\prime}$ to a sequence of the $e_{i}^{\prime \prime}$ relative to $y$, we have to make sure that $e_{3}^{\prime} \leq^{L} e_{3}^{\prime \prime}$ : But

$$
\begin{aligned}
& e_{3}^{\prime} \leq^{L} e_{3}^{\prime \prime \prime} \in y \text { for some } e_{3}^{\prime \prime \prime} \in y \text { since } y^{\prime} \sqsubseteq y, \text { and } \\
& e_{3}^{\prime \prime \prime}=e_{3}^{\prime \prime} \text { since } e_{2}^{\prime} \leq^{L} e_{3}^{\prime \prime}, e_{2}^{\prime} \leq^{L} e_{3}^{\prime \prime \prime}, \text { and } e_{3}^{\prime \prime}, e_{3}^{\prime \prime \prime} \in y
\end{aligned}
$$

Continuing in this way, we get:

$$
e_{0}^{\prime} \leq^{L} e_{1}^{\prime \prime} \geq^{R} e_{2}^{\prime \prime} \cdots \leq^{L} e_{2 i^{\prime}+1}^{\prime \prime}=e_{1} \geq^{R} e_{2} \cdots \leq^{L} e_{2 i+1}=e
$$


where $e_{2 i^{\prime}+1}^{\prime \prime}=e_{1}$ follows from Lemma 4 applied to $y, y, e_{2 i^{\prime}+1}^{\prime \prime}$, and $e_{1}$. Since $e_{2 j+1} \in y$ for all $0 \leq j \leq i$ and $e_{2 j+1}^{\prime \prime} \in y$ for all $0 \leq j \leq i^{\prime}$, by (2), we conclude that $x \sqsubseteq^{L} y$.

(4) Only the uniqueness of $z$ is in question, so suppose that $x \sqsubseteq^{L} z \sqsubseteq^{R} y$ and $x \sqsubseteq^{L} z^{\prime} \sqsubseteq^{R} y$. By symmetry, it is enough to show that $z \subseteq z^{\prime}$. So suppose that $e \in z$. Then by (2) there are $e_{0}$ in $x$ and $e_{1}$ in $z$ such that $e \preceq_{z} e_{1} \geq^{L} e_{0}$. We begin by showing that $e_{1} \in z^{\prime}$. Since $e_{0} \in x \sqsubseteq z^{\prime}$ there is an $e_{1}^{\prime}$ in $z^{\prime}$ such that $e_{0} \sqsubseteq^{L} e_{1}^{\prime}$. So $e_{1} \downarrow^{L} e_{1}^{\prime}$ and therefore, by Lemma 4 applied to $z, z^{\prime}, e_{1}$ and $e_{1}^{\prime}$, we have $e_{1}=e_{1}^{\prime} \in z^{\prime}$. Now by Lemma 5 applied to $z, z^{\prime}, e_{1}$, and $e$ we have $e \preceq_{z^{\prime}} e_{1}$ and so $e \in z^{\prime}$, concluding the proof.

Proposition 2 Let $E$ be a bistructure. The following properties hold of the associated biorder:

1. all $\sqsubseteq$-directed lubs and $\sqsubseteq^{R}$-bounded lubs exist,

2. all $\sqsubseteq^{R}$-lubs of $\sqsubseteq^{R}$-directed sets exist, coinciding with their $\sqsubseteq$-lubs, and

3. a configuration is $\sqsubseteq$-compact iff it is $\sqsubseteq^{R}$-compact iff it is finite.

It follows that $\left(\Gamma(E), \sqsubseteq^{R}\right)$ and $(\Gamma(E), \sqsubseteq)$ are $\omega$-algebraic cpos (with common least element $\emptyset)$, and that, moreover, $\left(\Gamma(E), \sqsubseteq^{R}\right)$ is bounded complete, i.e., is a Scott domain.

Proof. (1) Let $\Delta$ be $\sqsubseteq$-directed. We show:

$z=\left\{e \in \bigcup \Delta \mid e\right.$ is $\leq^{L}$-maximal in $\left.\bigcup \Delta\right\}$ is the $\sqsubseteq$-lub of $\Delta$

We first check that $z$ is a configuration. If $e_{1}, e_{2} \in z$, then $e_{1} \in \delta_{1}$ and $e_{2} \in \delta_{2}$ for some $\delta_{1}, \delta_{2}$ in $\Delta$. Let $\delta$ in $\Delta$ be such that $\delta_{1}, \delta_{2} \sqsubseteq \delta$. Then by definition of $z$ and $\sqsubseteq$, it follows that $e_{1}, e_{2} \in \delta$. Therefore $e_{1} \smile e_{2}$. If $e \in z$ and $e_{1} \leq{ }^{R} e$, let $\delta$ in $\Delta$ be such that $e \in \delta$. Since $\delta$ is secured, there exists $e_{2}$ in $\delta$ such that $e_{1} \leq^{L} e_{2}$. By definition of $z$ and by Axiom 2 (cf. Remark 1), we can find $e_{3}$ in $z$ such that $e_{2} \leq^{L} e_{3}$. Hence $z$ is indeed a configuration. It is obvious from the definition of $z$ that $\delta \sqsubseteq z$ holds for any $\delta$ in $\Delta$, and that if $z_{1}$ is an $\sqsubseteq$-upper bound of $\Delta$ then $z \sqsubseteq z_{1}$. 
The $\sqsubseteq^{R}$-bounded lubs exist: if $X \subseteq \Gamma(E)$ and if $x$ is an $\bigsqcup^{R}$-upper bound of $X$, then $\bigcup X$ is consistent as a subset of $x$ and secured as a union of secured sets of events.

(2) Let $\Delta$ be $\sqsubseteq^{R}$-directed (and hence a fortiori $\sqsubseteq$-directed). Clearly $\bigsqcup^{R} \Delta$ exists and is $\bigcup \Delta$; we prove that $\bigsqcup \Delta=\bigcup \Delta$ (where $\bigsqcup^{R}$ and $\bigsqcup$ are relative to $\sqsubseteq^{R}$ and $\sqsubseteq$, respectively). We have to show that any $e$ in $\bigcup \Delta$ is $\leq^{L}$-maximal. Suppose there exists $e_{1}$ in $\bigcup \Delta$ such that $e \leq^{L} e_{1}$. Then, applying Lemma 4 to $\bigcup \Delta, \bigcup \Delta, e$, and $e_{1}$, we get $e=e_{1}$.

(3) We prove three implications.

- $x$ finite $\Rightarrow x \sqsubseteq$-compact: Let $\left\{e_{1}, \ldots, e_{n}\right\} \sqsubseteq \bigsqcup \Delta$. There exist $e_{1}^{\prime}, \ldots, e_{n}^{\prime}$ in $\bigsqcup \Delta$ such that $e_{i} \leq^{L} e_{i}^{\prime}$ for all $i$. Let $\delta_{1}, \ldots \delta_{n}$ in $\Delta$ be such that $e_{i}^{\prime} \in \delta_{i}$ for all $i$, and let $\delta$ in $\Delta$ be such that $\delta_{i} \sqsubseteq \delta$ for all $i$. Then by the $\leq^{L}$-maximality of $e_{1}^{\prime}, \ldots, e_{n}^{\prime}$ we get that $e_{i}^{\prime} \in \delta$ for all $i$. Hence $\left\{e_{1}, \ldots, e_{n}\right\} \sqsubseteq \delta$.

- $x \sqsubseteq$-compact $\Rightarrow x \sqsubseteq^{R}$-compact: If $x \sqsubseteq^{R} \bigsqcup^{R} \Delta$, then, a fortiori, $x \sqsubseteq$ $\bigsqcup \bar{\Delta}$, therefore $x \sqsubseteq \bar{\delta}$ for some $\delta$ in $\Delta$. We show that actually $x \sqsubseteq^{R} \bar{\delta}$ holds. Suppose $e \in x$ and let $e_{1}$ in $\delta$ be such that $e \leq^{L} e_{1}$. Then we get $e=e_{1}$ by Lemma 4 applied to $x, \delta$, e, and $e_{1}$.

- $x \sqsubseteq^{R}$-compact $\Rightarrow x$ finite: We claim that, for any $z$ :

$$
\left\{y \mid y \text { finite configuration and } y \sqsubseteq^{R} z\right\}
$$

is $\bigsqcup^{R}$-directed and has $z$ as lub. The directedness is obvious. We have to check that $z \sqsubseteq^{R} \bigsqcup^{R}\left\{y \mid y\right.$ finite and $\left.y \sqsubseteq^{R} z\right\}$, i.e., for all $e$ in $z$, there exists a finite $y$ such that $y \sqsubseteq^{R} z$ and $e \in y$. The configuration $[e]_{z}$ (cf. Lemma 6) does the job.

Proposition 3 Let $E$ be a bistructure. Then the following properties hold:

1. the complete primes of $\left(\Gamma(E), \sqsubseteq^{R}\right)$ are the configurations of the form $[e]_{x}$, and

2. $\left(\Gamma(E), \sqsubseteq^{R}\right)$ is a dI-domain. 
Proof. (1) Consider a configuration $[e]_{x}$. We show it is a complete prime. If $Y$ is bounded above and $[e]_{x} \sqsubseteq^{R} \bigsqcup^{R} Y=\bigcup Y$, then $e \in y$ for some $y$ in $Y$. Since $[e]_{x} \uparrow^{R} y$, we infer that $[e]_{x} \subseteq y$, by Lemma 5. Conversely, every complete prime is of this form, since for any configuration $x$ we have $x=\bigcup\left\{[e]_{x} \mid e \in x\right\}$.

(2) A dI-domain is a Scott domain which is distributive (see Definition 7) and satisfies Axiom I, which states that a compact element dominates finitely many elements. Axiom I follows from the finiteness of compacts, proved in Proposition 2. Distributivity is then equivalent to prime-algebraicity, i.e., the property that any element is the lub of the complete primes that it dominates. (We refer to $[31,27]$ for a proof.) Prime-algebraicity is an immediate consequence of (1).

The properties proved in Proposition 2 and Proposition 3 correspond to the most interesting structure of Berry's bidomains. However, to show its configurations form a bidomain we will require a bistructure to fulfill extra axioms; these assure the existence of enough meets. We pursue these matters in Section 7.

\section{A category of bistructures}

Morphisms between bistructures correspond to configurations of the functionspace construction given below. They determine (certain - see Remark 4) extensional, linear ( $=$ stable and additive) functions on domains of configurations. Given bistructures $E_{i}=\left(E_{i}, \leq_{i}^{L}, \leq_{i}^{R}, \bigodot_{i}\right)$, for $i=0,1$, their linear function space is defined by:

$$
E_{0} \multimap E_{1}=\left(E_{0} \times E_{1}, \leq^{L}, \leq^{R}, \frown\right)
$$

where

$$
\begin{aligned}
& \left(e_{0}, e_{1}\right) \leq^{L}\left(e_{0}^{\prime}, e_{1}^{\prime}\right) \Leftrightarrow e_{0}^{\prime} \leq^{R} e_{0} \text { and } e_{1} \leq^{L} e_{1}^{\prime}, \\
& \left(e_{0}, e_{1}\right) \leq^{R}\left(e_{0}^{\prime}, e_{1}^{\prime}\right) \Leftrightarrow e_{0}^{\prime} \leq^{L} e_{0} \text { and } e_{1} \leq_{1}^{R} e_{1}^{\prime}
\end{aligned}
$$

and

$$
\left(e_{0}, e_{1}\right) \asymp\left(e_{0}^{\prime}, e_{1}^{\prime}\right) \Leftrightarrow e_{0} \bigodot_{0} e_{0}^{\prime} \text { and } e_{1} \smile_{1} e_{1}^{\prime} \text {. }
$$


It is straightforward to show that this is a bistructure. We define the category of bistructures BS by taking the morphisms from $E_{0}$ to $E_{1}$ to be configurations of $E_{0} \multimap E_{1}$, with composition being that of relations. We must show that this composition is well-defined and has identities.

Proposition 4 Let $\alpha$ be a configuration of $E \multimap E^{\prime}$ and $\beta$ be a configuration of $E^{\prime} \multimap E^{\prime \prime}$. Then their relational composition $\beta \circ \alpha$ is a configuration of $E \multimap E^{\prime \prime}$. Also the identity relation on a bistructure $E$ is a configuration of $E \multimap E$.

Proof. That identity relations are configurations relies, for securedness, on the factorisation property (1) of bistructures: if $\left(e_{1}, e_{2}\right) \leq_{R}(e, e)$, then $\left(e_{1}, e_{2}\right) \leq_{L}\left(e_{3}, e_{3}\right)$ where $e_{3}$ is obtained by factorisation of $e_{2} \leq e_{1}$. For the relational composition $\beta \circ \alpha$ to be a configuration we require it to be consistent and secured.

Consistency: From the definition of $\asymp$ on function space we require that for $\left(e_{1}, e_{1}^{\prime \prime}\right),\left(e_{2}, e_{2}^{\prime \prime}\right)$ in $\beta \circ \alpha$ that

$$
\text { (i) } e_{1} \frown e_{2} \Rightarrow e_{1}^{\prime \prime} \subsetneq e_{2}^{\prime \prime} \text { and }(i i) e_{1}^{\prime \prime} \asymp e_{2}^{\prime \prime} \Rightarrow e_{1} \asymp e_{2},
$$

facts which hold of the composition $\beta \circ \alpha$ because they hold of $\alpha$ and $\beta$.

Securedness: Suppose $\left(e, e^{\prime \prime}\right) \in \beta \circ \alpha$ and that

$$
\left(e_{0}, e_{0}^{\prime \prime}\right) \leq^{R}\left(e, e^{\prime \prime}\right)
$$

i.e., $e \leq^{L} e_{0}$ and $e_{0}^{\prime \prime} \leq^{R} e^{\prime \prime}$. It is required that there is

$$
\left(e^{\star}, e^{\prime \prime \star}\right) \in \beta \circ \alpha
$$

such that

$$
\left(e_{0}, e_{0}^{\prime \prime}\right) \leq^{L}\left(e^{\star}, e^{\prime \prime \star}\right),
$$

i.e., $e^{\star} \leq^{R} e_{0}$ and $e_{0}^{\prime \prime} \leq^{L} e^{\prime \prime \star}$. [In the following argument, it is helpful to refer to the diagram below.]

As $\left(e, e^{\prime \prime}\right) \in \beta \circ \alpha$ there is $e_{0}^{\prime \star}$ such that $\left(e, e_{0}^{\prime \star}\right) \in \alpha$ and $\left(e_{0}^{\prime \star}, e^{\prime \prime}\right) \in \beta$. Because $e_{0}^{\prime \prime} \leq^{R} e^{\prime \prime}$, we obtain that

$$
\left(e_{0}^{\star \star}, e_{0}^{\prime \prime}\right) \leq^{R}\left(e_{0}^{\prime \star}, e^{\prime \prime}\right)
$$


As $\beta$ is secured there is $\left(e_{1}^{\prime}, e_{1}^{\prime \prime}\right)$ in $\beta$ for which $\left(e_{0}^{\prime \star}, e_{0}^{\prime \prime}\right) \leq^{L}\left(e_{1}^{\prime}, e_{1}^{\prime \prime}\right)$, i.e.,

$$
\left(e_{1}^{\prime}, e_{1}^{\prime \prime}\right) \in \beta \text { and } e_{1}^{\prime} \leq^{R} e_{0}^{\prime \star} \text { and } e_{0}^{\prime \prime} \leq^{L} e_{1}^{\prime \prime} .
$$

As $e \leq^{L} e_{0}$ and $e_{1}^{\prime} \leq^{R} e_{0}^{\prime \star}$, we have $\left(e_{0}, e_{1}^{\prime}\right) \leq^{R}\left(e, e_{0}^{\prime \star}\right)$. But $\alpha$ is secured, so there is $\left(e_{1}, e_{1}^{\prime \star}\right)$ in $\alpha$ for which $\left(e_{0}, e_{1}^{\prime}\right) \leq^{L}\left(e_{1}, e_{1}^{\prime \star}\right)$, i.e.,

$$
\left(e_{1}, e_{1}^{\prime \star}\right) \in \alpha \text { and } e_{1} \leq^{R} e_{0} \text { and } e_{1}^{\prime} \leq^{L} e_{1}^{\prime \star} .
$$

From $(1 \alpha)$ and $(1 \beta)$ we obtain:

$$
e_{0} \geq^{R} e_{1} \quad\left(e_{1}, e_{1}^{\prime \star}\right) \in \alpha \quad e_{1}^{\prime \star} \geq_{L} e_{1}^{\prime} \quad\left(e_{1}^{\prime}, e_{1}^{\prime \prime}\right) \in \beta \quad e_{1}^{\prime \prime} \geq^{L} e_{0}^{\prime \prime}
$$

We now show that this pattern in $e_{1}, e_{1}^{\prime \star}, e_{1}^{\prime}, e_{1}^{\prime \prime}$, relative to $e_{0}$ and $e_{0}^{\prime \prime}$, must repeat.

It follows from $e_{1}^{\prime} \leq^{L} e_{1}^{\prime \star}$ that $\left(e_{1}^{\prime \star}, e_{1}^{\prime \prime}\right) \leq^{R}\left(e_{1}^{\prime}, e_{1}^{\prime \prime}\right) \in \beta$. As $\beta$ is secured, there is an $\left(e_{2}^{\prime}, e_{2}^{\prime \prime}\right)$ in $\beta$ for which $\left(e_{1}^{\prime \star}, e_{1}^{\prime \prime}\right) \leq^{L}\left(e_{2}^{\prime}, e_{2}^{\prime \prime}\right)$, i.e.,

$$
\left(e_{2}^{\prime}, e_{2}^{\prime \prime}\right) \in \beta \text { and } e_{2}^{\prime} \leq^{R} e_{1}^{\prime \star} \text { and } e_{1}^{\prime \prime} \leq^{L} e_{2}^{\prime \prime}
$$

As $e_{2}^{\prime} \leq^{R} e_{1}^{\prime \star}$, we have $\left(e_{1}, e_{2}^{\prime}\right) \leq^{R}\left(e_{1}, e_{1}^{\prime \star}\right) \in \alpha$. But $\alpha$ is secured, so there is an $\left(e_{2}, e_{2}^{\prime \star}\right)$ in $\alpha$ for which $\left(e_{1}, e_{2}^{\prime}\right) \leq^{L}\left(e_{2}, e_{2}^{\prime \star}\right)$, i.e.,

$$
\left(e_{2}, e_{2}^{\prime \star}\right) \in \alpha \text { and } e_{2} \leq^{R} e_{1} \text { and } e_{2}^{\prime} \leq^{L} e_{2}^{\prime \star}
$$

and the pattern in (1) repeats in (2) below-obtained directly from $(2 \alpha)$ and $(2 \beta)$ :

$$
e_{0} \geq^{R} e_{2} \quad\left(e_{2}, e_{2}^{\prime \star}\right) \in \alpha \quad e_{2}^{\prime \star} \geq_{L} e_{2}^{\prime} \quad\left(e_{2}^{\prime}, e_{2}^{\prime \prime}\right) \in \beta \quad e_{2}^{\prime \prime} \geq^{L} e_{0}^{\prime \prime}
$$

where $e_{2}^{\prime} \leq^{R} e_{1}^{\prime \star}$. This can be repeated infinitely. Diagrammatically:

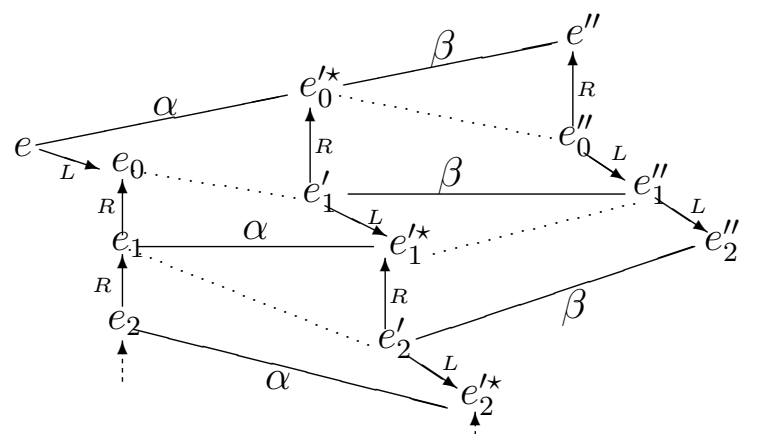


The chain

$$
e_{0}^{\prime \star} \geq^{R} e_{1}^{\prime} \leq^{L} e_{1}^{\star \star} \geq^{R} e_{2}^{\prime} \leq^{L} e_{2}^{\prime \star} \geq^{R} \cdots
$$

must eventually be constant by Axiom 2(a) on bistructures. Hence we obtain

$$
\left(e_{n}, e_{n}^{\star \star}\right) \in \alpha \text { and } e_{n}^{\prime}=e_{n}^{\star \star} \text { and }\left(e_{n}^{\prime}, e_{n}^{\prime \prime}\right) \in \beta
$$

yielding $\left(e_{n}, e_{n}^{\prime \prime}\right)$ in $\beta \circ \alpha$ with $e_{n} \leq^{R} e_{0}$ and $e_{0}^{\prime \prime} \leq^{L} e_{n}^{\prime \prime}$, i.e., $\left(e_{0}, e_{0}^{\prime \prime}\right) \leq^{L}\left(e_{n}, e_{n}^{\prime \prime}\right)$; so $\left(e_{n}, e_{n}^{\prime \prime}\right)$ fulfills the requirements we seek for $\left(e^{\star}, e^{\prime \prime \star}\right)$.

Proposition 5 Let $F$ be a configuration of $E \multimap E^{\prime}$ and let $x$ be a configuration of $E$. Defining

$$
F \cdot x=\left\{e^{\prime} \mid \exists e \in x \quad\left(e, e^{\prime}\right) \in F\right\}
$$

yields a configuration of $E^{\prime}$. The function $x \mapsto F \cdot x: \Gamma(E) \rightarrow \Gamma\left(E^{\prime}\right)$ is linear with respect to $\bigsqcup^{R}$ and continuous with respect to $\sqsubseteq$.

Proof. The first part of the statement is a consequence of Proposition 4, since $F \cdot x$ can be read as $F \circ x$ if $x$ is viewed as a configuration from $I$ (the tensor unit, defined at the beginning of the next section) to $E$. The verification that $x \mapsto F \cdot x$ is linear is easy. We check only that it preserves compatible binary lubs. Suppose that $e^{\prime} \in F \cdot\left(x \sqcup^{R} y\right)$, i.e., $\left(e, e^{\prime}\right) \in F$ for some $e$ in $x \sqcup^{R} y$. Then $e \in x$ or $e \in y$, hence $e^{\prime} \in(F \cdot x) \sqcup^{R}(F \cdot y)$.

For continuity, by Lemma 7 below, it is enough to check $\sqsubseteq$-monotonicity, which is proved using a "staircase argument" similar to that of the previous proof: if $x \sqsubseteq y$ and $e^{\prime} \in F \cdot x$, let $e$ in $x$ be such that $\left(e, e^{\prime}\right) \in F \cdot x$, and let $e_{1}$ in $y$ be such that $e \leq^{L} e_{1}$. Since $\left(e_{1}, e^{\prime}\right) \leq^{R}\left(e, e^{\prime}\right)$, there exists $\left(e_{2}, e_{1}^{\prime}\right)$ in $F$ such that $e^{\prime} \leq^{L} e_{1}^{\prime}$ and $e_{2} \leq^{R} e_{1}$. From there we find an $e_{3}$ such that $e_{3} \in y$ and $e_{2} \leq^{L} e_{3}$, which leads to an $\left(e_{4}, e_{2}^{\prime}\right)$ in $F$ such that $\left(e_{3}, e_{1}^{\prime}\right) \leq^{L}\left(e_{4}, e_{2}^{\prime}\right)$. In this way, we construct $e^{\prime} \leq^{L} e_{1}^{\prime} \leq^{L} e_{2}^{\prime} \cdots$, where the $e_{i}^{\prime}$ are in $y$. The sequence eventually ends, yielding an $e_{i}^{\prime}$ that fits.

Lemma 7 Let $E$ and $E^{\prime}$ be bistructures. Suppose that $f: \Gamma(E) \rightarrow \Gamma\left(E^{\prime}\right)$ is $\sqsubseteq^{R}$-continuous and $\sqsubseteq$-monotonic. Then it is also $\sqsubseteq$-continuous.

Proof. Suppose $\left\{e_{1}, \ldots, e_{n}\right\} \sqsubseteq f(x)$. Then there exist $e_{1}^{\prime}, \ldots, e_{n}^{\prime}$ in $f(x)$ such that $e_{i} \leq^{L} e_{i}^{\prime}$ for all $i$. By $\bigsqcup^{R}$-continuity, there exists a finite $x_{1} \sqsubseteq^{R} x$ such that $e_{1}^{\prime}, \ldots, e_{n}^{\prime} \in f\left(x_{1}\right)$, hence $\left\{e_{1}, \ldots, e_{n}\right\} \sqsubseteq f\left(x_{1}\right)$. 
Remark 4 Not all $\sqsubseteq^{R}$-linear and $\sqsubseteq$-continuous functions are represented by a morphism. The represented functions also satisfy a uniformity requirement, where $a \sqsubseteq^{R}$-stable and $\sqsubseteq$-continuous function $f$ from $\Gamma(E)$ to $\Gamma\left(E^{\prime}\right)$ is uniform iff for all $e$ in $E$ and configurations $x$ containing $e$ and for all $e^{\prime}$ in $E^{\prime}$ we have that $\left([e]_{x}, e^{\prime}\right) \in \operatorname{tr}(f)$ implies $\left([e], e^{\prime}\right) \in \operatorname{tr}(f)$ (see Definition 6 below, and cf. Remark 3); unfortunately, even this condition is not sufficient for representability. On a more positive note, one can show that the assignment of functions to configurations is 1-1.

\section{A model of classical linear logic}

Here we give the constructions showing that BS is a (non-compactly closed) model of classical linear logic. The constructions extend those of Girard (recalled in Section 2). Define linear negation, the involution of linear logic, by

$$
E^{\perp}=\left(E, \geq^{R}, \geq^{L}, \asymp\right)
$$

where $E=\left(E, \leq^{L}, \leq^{R}, \bigodot\right)$. Clearly $\left(E^{\perp}\right)^{\perp}=E$. The remaining multiplicatives, $\wp($ par $)$ and $\otimes$ (tensor), are determined by the usual isomorphisms of classical linear logic:

$$
E_{0} \wp E_{1} \cong\left(E_{0}^{\perp} \multimap E_{1}\right), \quad E_{0} \otimes E_{1} \cong\left(E_{0} \multimap E_{1}^{\perp}\right)^{\perp}
$$

In more detail, the tensor product is defined as follows:

$$
E_{0} \otimes E_{1}=\left(E_{0} \times E_{1}, \leq^{L}, \leq^{R}, \frown\right)
$$

where

$$
\begin{aligned}
& \left(e_{0}, e_{1}\right) \leq^{L}\left(e_{0}^{\prime}, e_{1}^{\prime}\right) \Leftrightarrow e_{0} \leq^{L} e_{0}^{\prime} \text { and } e_{1} \leq^{L} e_{1}^{\prime}, \\
& \left(e_{0}, e_{1}\right) \leq^{R}\left(e_{0}^{\prime}, e_{1}^{\prime}\right) \Leftrightarrow e_{0} \leq^{R} e_{0}^{\prime} \text { and } e_{1} \leq_{1}^{R} e_{1}^{\prime}
\end{aligned}
$$

and

$$
\left(e_{0}, e_{1}\right) \subsetneq\left(e_{0}^{\prime}, e_{1}^{\prime}\right) \Leftrightarrow e_{0} \bigodot_{0} e_{0}^{\prime} \text { and } e_{1} \bigodot_{1} e_{1}^{\prime}
$$

The construction $E^{\perp}$ is isomorphic to $(E \multimap I)$ where $I=(\{\bullet\}, i d, i d, i d)$ is the unit of $\otimes$. Product and coproduct in the category BS are (again) obtained by disjoint juxtaposition (now of bistructures), extending conflict 
across the two event sets in the case of coproduct. The terminal object is $1=(\emptyset, \emptyset, \emptyset, \emptyset)$.

We define the exponential $! E$ of a bistructure $E$ by:

$$
! E=\left(\Gamma(E)^{0}, \sqsubseteq^{L}, \sqsubseteq^{R}, \uparrow^{R}\right)
$$

(recall $\sqsubseteq^{L}$ and $\sqsubseteq^{R}$, and $\uparrow^{R}$ from Definition 3 ).

Lemma $8 ! E$ is a bistructure.

Proof. Obviously, $\sqsubseteq^{R}$ is a partial order. By Proposition $1, \sqsubseteq^{L}$ is a partial order and so Axiom 1 is verified. Given the definition of $\bigodot_{!}$, Axiom 3(b) holds a fortiori, and we can rephrase Axiom 3(a) as:

$$
\left(x_{1} \downarrow^{L} x_{2} \text { and } x_{1} \uparrow^{R} x_{2}\right) \Rightarrow x_{1}=x_{2}
$$

which is an immediate consequence of Proposition 1.4.

The main difficulty in the proof is in showing Axiom 2(b), i.e., that the relation $\preceq !=\left(\sqsupseteq^{L} \cup \sqsubseteq^{R}\right)^{\star}$ of $! E$ is a partial order. We need only show antisymmetry. Thus suppose for $x_{i}, x_{i}^{\prime}$ in $\Gamma(E)^{0}$ we have:

$$
x_{0} \sqsubseteq^{R} x_{0}^{\prime} \sqsupseteq^{L} x_{1} \sqsubseteq^{R} x_{1}^{\prime} \sqsupseteq^{L} \cdots \sqsupseteq^{L} x_{n} \sqsubseteq^{R} x_{n}^{\prime}
$$

with $x_{n}=x_{0}$ and $x_{n}^{\prime}=x_{0}^{\prime}$. We shall show $x_{i}=x_{i}^{\prime}=x_{j}=x_{j}^{\prime}$ for all $i, j$. Then by the definition of $\preceq$ ! on ! $E$ it follows that $\preceq$ ! is antisymmetric.

Define $f i x=\bigcap_{i} x_{i}$. We first show $f i x \in \Gamma(E)$. Consistency is obvious. Suppose $e \in f i x$ and $e_{1} \leq^{R} e$. Since $e \in x_{i}$, there exist $e_{1}^{i}$ in $x_{i}$ such that $e_{1} \leq^{L} e_{1}^{i}$, for all $i \geq 1$. Since $x_{i} \sqsubseteq x_{i-1}^{\prime}$, there exist $e_{1}^{i i}$ in $x_{i-1}^{\prime}$ such that $e_{1}^{i} \leq^{L} e_{1}^{i}$, for all $i \geq 1$. We now apply Lemma 4 twice.

- From $e_{1} \leq^{L} e_{1}^{i}, e_{1} \leq^{L} e_{1}^{i-1}$ and $x_{i-1} \sqsubseteq^{R} x_{i-1}^{\prime}$ we conclude $e_{1}^{i i}=e_{1}^{i-1}$, therefore $e_{1}^{i-1} \geq^{L} e_{1}^{i}$.

- From $e_{1} \leq^{L} e_{1}^{0}, e_{1} \leq^{L} e_{1}^{n}$ and $x_{0}=x_{n}$, we obtain $e_{1}^{0}=e_{1}^{n}$.

Since $\leq^{L}$ is a partial order, we get: $e_{1}^{0}=\cdots=e_{1}^{i}=\cdots=e_{1}^{n}$, hence $e_{1}^{0} \in f i x$, which shows that $f i x$ is secured. Consequently $f i x \in \Gamma(E)$ and clearly $f i x \sqsubseteq^{R} x_{i}, x_{i}^{\prime}$ for all $i$. It remains to show $f i x=x_{i}=x_{i}^{\prime}$ for all $i$. 
Notice that in chain (1) we can bring any index $i$ to the head position, by a circular permutation. Thus, it suffices to show $x_{0}=x_{0}^{\prime}=f i x$.

Take $e$ in $x_{0}^{\prime}$. Then by repeated use of Proposition 1, we deduce from (1) that

$$
e=e_{0} \preceq_{x_{0}^{\prime}} e_{0}^{\prime} \geq^{L} e_{1} \preceq_{x_{1}^{\prime}} e_{1}^{\prime} \geq^{L} e_{2} \cdots \geq^{L} e_{m} \preceq_{x_{[m]_{n}}^{\prime}} e_{m}^{\prime} \geq^{L} e_{m+1} \cdots
$$

for some $e_{i}$ in $x_{[i]_{n}}$ and $e_{i}^{\prime}$ in $x_{[i]_{n}}^{\prime}$ where $i \in \omega$ (here $[m]_{n}$ is $m$ modulo $n$ ).

The sequence has been continued infinitely by going around and around the loop (1). As $x_{0}^{\prime}$ is finite and the sequence (2) visits $x_{0}^{\prime}$ infinitely often there must be $e_{m}, e_{q}$ in $x_{0}^{\prime}$ such that $m<q$ and $[m]_{n}=[q]_{n}=0$ and $e_{m}=e_{q}$. Then as $\preceq$ is a partial order, $e_{m}=e_{m}^{\prime}=e_{m+1}=\cdots=e_{q}$. Thus $e_{m} \in$ fix so the sequence (2) eventually contains an element of fix. We know fix $\bigsqcup^{R} x_{i}, x_{i}^{\prime}$, for all $i$. Now, working backwards along the chain (2), starting at $e_{m}$, we show that all elements of the chain are in fix.

- From $e_{i+1}$ to $e_{i}^{\prime}$ : this follows from Lemma 4 applied to $f i x, x_{i}^{\prime}, e_{i+1}$, and $e_{i}^{\prime}$.

- From $e_{i}^{\prime}$ to $e_{i}$ : by Lemma 5 .

In particular, $e_{0}(=e) \in f i x$. We have proved $x_{0}^{\prime} \sqsubseteq^{R} f i x$, which together with fix $\sqsubseteq^{R} x_{0} \sqsubseteq^{R} x_{0}^{\prime}$ implies $x_{0}=x_{0}^{\prime}=$ fix as required. Thus the relation $\preceq_{\text {! on }}$ $! E$ is a partial order.

Finally, we prove Axiom 2(a), i.e., $\left\{x^{\prime} \in \Gamma(E)^{0} \mid x^{\prime} \preceq ! x\right\}$ is finite, for $x \in \Gamma(E)^{0}$. By Proposition 1 it is clear that

$$
x^{\prime} \preceq ! x \Rightarrow \forall e^{\prime} \in x^{\prime} \exists e \in x \quad e^{\prime} \preceq e
$$

Thus:

$$
x^{\prime} \preceq ! x \Rightarrow x^{\prime} \subseteq \bigcup\left\{e^{\prime} \mid \exists e \in x \quad e^{\prime} \preceq e\right\}
$$

As $x$ is finite and $\preceq$ is finitary, we have $\left\{x^{\prime} \in \Gamma(E)^{0} \mid x^{\prime} \preceq\right.$ ! $\left.x\right\}$ is finite, as required.

The configurations of the bistructure $! E_{0} \multimap E_{1}$ are in 1-1 correspondence with the functions from $\left(\Gamma\left(E_{0}\right), \sqsubseteq^{R}, \sqsubseteq\right)$ to $\left(\Gamma\left(E_{1}\right), \sqsubseteq^{R}, \sqsubseteq\right)$ considered by Berry in his cartesian closed category of bidomains. We need notions of trace and extensional and stable orderings for such functions (cf. Section 3): 
Definition 6 Let $f$ be $a \sqsubseteq$-continuous and $\sqsubseteq^{R}$-stable function from $\Gamma\left(E_{0}\right)$ to $\Gamma\left(E_{1}\right)$. Then:

$$
\operatorname{tr}(f)=\left\{(x, e) \in \Gamma\left(E_{0}\right)^{0} \times E_{1} \mid x \text { is } \bigsqcup^{R} \text {-minimal such that } e \in f(x)\right\}
$$

The stable ordering on such functions is defined by:

$$
f \leq g \Leftrightarrow \forall x \sqsubseteq^{R} \text { y } f(x)=f(y) \cap g(x)
$$

The extensional ordering on such functions is defined by:

$$
f \sqsubseteq g \Leftrightarrow \forall x \quad f(x) \sqsubseteq g(x)
$$

Proposition 6 Let $E_{0}, E_{1}$ be bistructures. For $R$ in $\Gamma\left(! E_{0} \multimap E_{1}\right)$ and $x$ in $\Gamma\left(E_{0}\right)$ define

$$
\bar{R}(x)=\left\{e \mid \exists x_{0} \sqsubseteq^{R} x\left(x_{0}, e\right) \in R\right\} .
$$

Then $\bar{R}$ is a function $\Gamma\left(E_{0}\right) \rightarrow \Gamma\left(E_{1}\right)$ which is continuous with respect to $\sqsubseteq$ and stable with respect to $\bigsqcup^{R}$ on configurations. In fact, $R \mapsto \bar{R}$ is a 1-1 correspondence between configurations of $! E_{0} \multimap E_{1}$ and such functions, with inverse $f \mapsto \operatorname{tr}(f)$. Further, for the stable ordering of functions we have:

$$
f \leq g \Leftrightarrow \operatorname{tr}(f) \sqsubseteq^{R} \operatorname{tr}(g)
$$

and for the extensional ordering we have:

$$
f \sqsubseteq g \Leftrightarrow \operatorname{tr}(f) \sqsubseteq \operatorname{tr}(g)
$$

Proof. We first check that $\bar{R}(x)$ is a configuration. Consistency follows from the definition of $\subsetneq$ in $! E_{0} \multimap E_{1}$. Suppose $e \in \bar{R}(x)$ and $e^{\prime} \leq^{R} e$. Choose $x_{0} \sqsubseteq^{R} x$ such that $\left(x_{0}, e\right) \in R$. Then $\left(x_{0}, e^{\prime}\right) \leq^{R}\left(x_{0}, e\right)$, hence by securedness there exists $\left(x^{\prime \prime}, e^{\prime \prime}\right)$ in $R$ such that $\left(x_{0}, e^{\prime}\right) \leq^{L}\left(x^{\prime \prime}, e^{\prime \prime}\right)$. Then $e^{\prime \prime}$ fits since $x^{\prime \prime} \sqsubseteq^{R} x_{0} \sqsubseteq^{R} x$. Thus the function $\bar{R}$ is well-defined. It is $\bigsqcup^{R}$-continuous by construction and $\sqsubseteq^{R}$-stable by the definition of $\subsetneq$ in $! E_{0} \multimap E_{1}$. We next show that $\bar{R}$ is $\sqsubseteq$-monotonic, hence $\sqsubseteq$-continuous, by Lemma 7 . Suppose $x \sqsubseteq y$ and $e \in \bar{R}(x)$. Choose $x_{0} \sqsubseteq^{R} x$ such that $\left(x_{0}, e\right) \in R$. Let us factorise $x_{0} \sqsubseteq y$ as $x_{0} \sqsubseteq^{L} x_{1} \sqsubseteq^{R} y$. Since $\left(x_{1}, e\right) \leq^{R}\left(x_{0}, e\right)$, there exists $\left(x_{2}, e_{1}\right)$ in $R$ such that $\left(x_{1}, e\right) \leq^{L}\left(x_{2}, e_{1}\right)$, by securedness. Then $e_{1}$ fits, since $e \leq^{L} e_{1}$ and $x_{2} \sqsubseteq^{R} x_{1} \sqsubseteq^{R} y$. 
We now show that tr is the inverse of $R \mapsto \bar{R}$. Suppose $f: \Gamma\left(E_{0}\right) \rightarrow \Gamma\left(E_{1}\right)$ is $\sqsubseteq$-continuous and $\sqsubseteq^{R}$-stable. We need first that $\operatorname{tr}(f) \in \Gamma\left(! E_{0} \multimap E_{1}\right)$, i.e., that $\operatorname{tr}(f)$ is consistent and secured:

Consistency: Suppose $(x, e),\left(x^{\prime}, e^{\prime}\right) \in \operatorname{tr}(f)$ and that $(x, e) \asymp\left(x^{\prime}, e^{\prime}\right)$, i.e., $x \uparrow^{R} x^{\prime}$ and $e \asymp e^{\prime}$. We show $(x, e)=\left(x^{\prime}, e^{\prime}\right)$. As $e, e^{\prime} \in f\left(x \cup x^{\prime}\right)$, we must have $e \asymp e^{\prime}$, which combined with $e \asymp e^{\prime}$, entails $e=e^{\prime}$. Now, $(x, e),\left(x^{\prime}, e\right)$ are both in the trace of $f$. Because $x \uparrow^{R} x^{\prime}$ and $f$ is $\sqsubseteq^{R}$-stable we conclude that $x=x^{\prime}$.

Securedness: Suppose $\left(x^{\prime}, e^{\prime}\right) \leq^{R}(x, e) \in \operatorname{tr}(f)$. Then $x \sqsubseteq^{L} x^{\prime}$ and $e^{\prime} \leq^{R} e$. As $f$ is $\sqsubseteq$-monotonic, $f(x) \sqsubseteq f\left(x^{\prime}\right)$. Because $e^{\prime} \leq^{R} e$ and $e \in f(x)$, we see that $e^{\prime}$ is in the $\leq$-downwards-closure of $f\left(x^{\prime}\right)$. Thus by Lemma 3 , there exists $e^{\prime \prime}$ in $f\left(x^{\prime}\right)$ such that:

$$
e^{\prime} \leq^{L} e^{\prime \prime}
$$

By the definition of $\operatorname{tr}(f)$, there is

$$
x_{0} \sqsubseteq^{R} x^{\prime}
$$

such that

$$
\left(x_{0}, e^{\prime \prime}\right) \in \operatorname{tr}(f)
$$

Combining (1), (2), (3) we obtain, as required

$$
\left(x^{\prime}, e^{\prime}\right) \leq^{L}\left(x_{0}, e^{\prime \prime}\right) \in \operatorname{tr}(f) .
$$

For $f$ a $\sqsubseteq$-continuous, $\sqsubseteq^{R}$-stable function $\Gamma\left(E_{0}\right) \rightarrow \Gamma\left(E_{1}\right)$ its continuity with respect to $\sqsubseteq^{R}$ entails $\overline{\operatorname{tr}(f)}=f$. For $R$ in $\Gamma\left(! E_{0} \multimap E_{1}\right)$ a direct translation of the definitions yields $\operatorname{tr}(\bar{R})=R$. Thus the map $R \mapsto \bar{R}=R$ is a 1-1 correspondence.

Turning to the two orderings of functions, the claim for the stable ordering is established exactly as in the case of webs [11]. For the extensional ordering, suppose first that $f \sqsubseteq g$ and choose $(x, e)$ in $\operatorname{tr}(f)$. Then $e \in f(x) \sqsubseteq g(x)$, and so for some $e^{\prime} \geq^{L} e, e^{\prime} \in g(x)$. But then there is an $x_{0} \sqsubseteq^{R} x$ such that $\left(x_{0}, e^{\prime}\right) \in \operatorname{tr}(g)$ and we have that $(x, e) \leq^{L}\left(x_{0}, e^{\prime}\right) \in \operatorname{tr}(g)$. Conversely, suppose that $\operatorname{tr}(f) \sqsubseteq \operatorname{tr}(g)$ and choose $e, x$ with $e \in f(x)$. Then for some subset $x_{0}$ of $x,\left(x_{0}, e\right) \in \operatorname{tr}(f) \sqsubseteq \operatorname{tr}(g)$, and so there is a $y \sqsubseteq^{R} x_{0}$ and an $e^{\prime} \geq^{L} e$ such that $\left(y, e^{\prime}\right) \in \operatorname{tr}(g)$. Then we have $e \leq^{L} e^{\prime} \in g(y) \sqsubseteq^{R} g(x)$, concluding the proof. 
This section has provided the key constructions for showing that BS is a model of classical linear logic, and that the associated co-Kleisli category is equivalent to one of biorders:

Theorem 1 The category BS forms a linear category in the sense of [24]. The exponential! forms a comonad on the category BS. Together they form a model of classical linear logic (a Girard category in the sense of [24]-see also [4]).

The associated co-Kleisli category is (necessarily) cartesian closed and isomorphic to the category whose objects are the structures $\left(\Gamma(E), \sqsubseteq^{R}, \sqsubseteq\right)$, where $E$ is a bistructure, and whose morphisms are the $\sqsubseteq$-continuous and $\sqsubseteq^{R}$-stable functions.

PROOF. The proof that BS forms a linear category is a straightforward extension of the web case, while the facts about the co-Kleisli category largely follow from Proposition 6. For the monoidal structure, at the level of events, the canonical isomorphisms are given by:

$$
\begin{aligned}
\left(\left(e_{0}, e_{1}\right), e_{2}\right) & \leftrightarrow\left(e_{0},\left(e_{1}, e_{2}\right)\right), \\
(e, \bullet) & \leftrightarrow e, \\
(\bullet, e) & \leftrightarrow e
\end{aligned}
$$

The first of these correspondences also serves to establish the closed structure. For example, we have:

$$
\begin{aligned}
& \left(\left(e_{0}, e_{1}\right), e_{2}\right) \leq_{E_{0} \otimes E_{1} \multimap E_{2}}^{L}\left(\left(e_{0}^{\prime}, e_{1}^{\prime}\right), e_{2}^{\prime}\right) \\
& \quad \Leftrightarrow e_{0}^{\prime} \leq_{E_{0}}^{R} e_{0} \text { and } e_{1}^{\prime} \leq_{E_{1}}^{R} e_{1} \text { and } e_{2} \leq_{E_{2}}^{L} e_{2}^{\prime} \\
& \quad \Leftrightarrow\left(e_{0},\left(e_{1}, e_{2}\right)\right) \leq_{E_{0} \multimap\left(E_{1} \multimap E_{2}\right)}^{L}\left(e_{0}^{\prime},\left(e_{1}^{\prime}, e_{2}^{\prime}\right)\right)
\end{aligned}
$$

The trace of the canonical morphism from $E$ to $(E \multimap I) \multimap I$ is:

$$
\{(e,((e, \bullet), \bullet)) \mid e \in E\}
$$

It is clear that it has as inverse the function whose trace is:

$$
\{(((e, \bullet), \bullet), e) \mid e \in E\}
$$

Turning to the comonadic structure, the counit $\varepsilon: ! E \multimap E$ is given by:

$$
\varepsilon=\left\{\left([e]_{x}, e\right) \mid e \in x \in \Gamma(E)\right\}
$$


For any $\alpha: ! E_{0} \multimap E_{1}$, its lift $\alpha^{\sharp}: ! E_{0} \multimap ! E_{1}$ is given by:

$$
\alpha^{\sharp}=\left\{\left(\bigcup_{i=1, n} x_{i},\left\{e_{1}, \ldots, e_{n}\right\}\right) \in ! E_{0} \times ! E_{1} \mid\left(x_{i}, e_{i}\right) \in \alpha(\text { for } i=1, n)\right\}
$$

(Then the comultiplication $\delta: ! E \multimap ! ! E$ is $\left(i d_{! E}\right)^{\sharp}$ and the functorial action of ! is $f \mapsto(f \circ \varepsilon)^{\sharp}$.) The canonical isomorphism ! $\left(E_{0} \times E_{1}\right) \cong\left(! E_{0}\right) \otimes\left(! E_{1}\right)$ is given by:

$$
x \leftrightarrow\left(x \cap E_{0}, x \cap E_{1}\right)
$$

The isomorphism $! 1 \cong I$ follows immediately from the observation that $\Gamma(1)$ is a singleton.

For the last part, the isomorphism is to send a bistructure $E$ to the biorder $\left(\Gamma(E), \sqsubseteq^{R}, \sqsubseteq\right)$, and a morphism $R: ! E_{0} \multimap E_{1}$ to the function $\bar{R}: \Gamma\left(E_{0}\right) \rightarrow \Gamma\left(E_{1}\right)$. Given the results of Proposition 6 , it only remains to show functoriality, and that is automatic from the comonadicity of the exponential, once we notice that $\bar{R}(x)=R \circ x^{\sharp}$, identifying elements of $\Gamma(E)$ with morphisms from !1 to $E$.

A fortiori, bistructures provide a model of simply-typed $\lambda$-calculi, and of PCF in particular. One interprets base types as event structures, i.e., bistructures of the form $(E, i d, \leq, \subsetneq)$. At first order, $\Gamma\left(! E_{0} \multimap E_{1}\right)$ is up to bijection the set of stable functions from $\Gamma\left(E_{0}\right)$ to $\Gamma\left(E_{1}\right)$, represented, not as an event structure, but as a bistructure with a non-trivial $\leq^{L}$-order which captures the extensional order between stable functions. At higher orders, as discussed in Section 2, the model diverges from the stable model by enforcing both $\sqsubseteq^{R}$-stability and $\sqsubseteq$-continuity of the morphisms. In the next section we shall see that this model coincides with that obtained from Berry's category of bidomains.

\section{Bidomains}

Bidomains are not very widely known so we present their definition here. They were introduced in Gérard Berry's Thèse de Doctorat d'Etat [2]. More details and background motivation can be obtained either from Berry's thesis or the references $[3,25]$.

The most general biordered domains Berry considered were the bicpos. 
Definition $7 A$ bicpo is a biorder $(D, \leq, \sqsubseteq)$ such that:

1. the structure $(D, \sqsubseteq)$ is a cpo with a continuous greatest-lower-bound operation $\sqcap$ (the order $\sqsubseteq$ is called the extensional order),

2. the structure $(D, \leq)$ is a cpo, with the same least element as $(D, \sqsubseteq)$ and the identity on $D$ is a continuous function from $(D, \leq)$ to $(D, \sqsubseteq)$ (the order $\leq$ is called the stable order),

3. the operation $\Pi$ is $\leq$-continuous - in fact, $\leq$-monotonicity suffices, by 1. and 2. (it follows that the $\leq$-greatest lower bound, $x \wedge y$, of points $x, y$, bounded above in $(D, \leq)$, exists and coincides with $x \sqcap y)$, and

4. for all $\sqsubseteq$-directed subsets $S, S^{\prime} \subseteq D$, if for all $s$ in $S, s^{\prime}$ in $S^{\prime}$ there are $t$ in $S, t^{\prime}$ in $S^{\prime}$ such that

$$
s \sqsubseteq t \text { and } s^{\prime} \sqsubseteq t^{\prime} \text { and } t \leq t^{\prime}
$$

then $\bigsqcup S \leq \bigsqcup S^{\prime}$.

A bicpo is distributive iff

- whenever $x, y$ are bounded above in $(D, \leq)$ then their least upper bounds, $x \vee y$, in $(D, \leq)$, and $x \sqcup y$, in $(D, \sqsubseteq)$, exist and coincide, and

- the cpo $(D, \leq)$ is distributive, i.e., for all $x, y, z$ in $D$, if $\{x, y, z\}$ is bounded above, then $x \wedge(y \vee z)=(x \wedge y) \vee(x \wedge z)$.

Bicpos form a category where the morphisms are taken to be those functions which are continuous with respect to the extensional order and conditionally multiplicative with respect to the stable order, i.e., the functions preserve binary compatible glbs. This category is cartesian closed; products are given by cartesian products of the underlying sets, the two orders being obtained in a coordinatewise fashion; the function space $\left[D_{0} \rightarrow D_{1}\right]$ consists of the set of morphisms from $D_{0}$ to $D_{1}$ with the extensional order $\sqsubseteq$ being the pointwise order, based on the extensional order of $D_{1}$, and with the order $\leq$ being the stable order of functions, based on the stable orders of $D_{0}$ and $D_{1}$.

Here are brief hints on how the axioms of Definition 7 are used. Lubs and glbs in the function spaces are defined pointwise. The continuity properties 
of the meet operation are put to use when proving that the pointwise lubs are conditionally multiplicative. Axiom 4 serves to establish that the pointwise extensional lubs are monotonic with respect to the stable ordering.

The smallest category of biordered domains Berry considered was that of bidomains. They are a form of distributive bicpo, satisfying a restriction which ensures that they are dI-domains with respect to the stable order.

Definition $8 A$ finite projection on a bicpo $D$ is a morphism $\psi: D \rightarrow D$ such that $h \circ h=h$ and $h(x) \leq x$, for all $x$ in $D$, and which is compact with respect to the stable order on $[D \rightarrow D]$.

A bicpo $D$ is said to be a bidomain iff it is distributive and there is a sequence of finite projections $\psi_{i}(i \in \omega)$ on it, increasing with respect to the stable order, whose least upper bound is the identity function $1_{D}: D \rightarrow D$.

The category of bidomains and of $\sqsubseteq$-continuous and $\leq$-stable functions is a full sub-cartesian closed category of that of bicpos. [In the subcategory, we have stable $=$ conditionally multiplicative.]

We now make good our earlier claim that configurations of (suitably restricted) bistructures form a bidomain. Let $E$ be a bistructure. The problem is that the axioms of bistructures adopted here are too weak to ensure the existence of enough $\sqsubseteq$-glbs to yield a bicpo. We can prove the existence of the required glbs provided we add two further axioms on bistructures:

Definition 9 A B-bistructure ("B" stands for "Bidomain") is a bistructure $\left(E, \leq^{L}, \leq^{R}, \smile\right)$ that satisfies the following two additional axioms:

4. if $e \geq^{L} e^{\prime}$ and $e \frown e^{\prime \prime}$, then $e^{\prime} \frown e^{\prime \prime}$, and

5. bounded binary $\leq^{L}$-lubs exist, i.e., if $e_{1}, e_{2} \leq^{L}$ e, for some event $e$, then the lub, $e_{1} \vee^{L} e_{2}$, with respect to $\leq^{L}$ exists.

B-bistructures are quite similar to the original version of bistructures in [25] - for example, Axioms 1, 2 and 5 are common, factorisation is unique in both, and all the other axioms relate to the interaction between coherence and the two partial orders. Axiom 3(a) is missing in [25]; instead, configurations are defined in a way equivalent to requiring for any elements $e, e^{\prime}$ that not only $e \subsetneq e^{\prime}$ but also $\neg\left(e \downarrow^{L} e^{\prime}\right)$. Pleasantly, as is straightforward to show, the class of biorders obtained from the original version is included in that obtained from B-bistructures. 
Proposition 7 Let $E$ be a B-bistructure. Then $\left(\Gamma(E), \sqsubseteq^{R}, \sqsubseteq\right)$ is a bidomain.

Proof. We must first show that $\left(\Gamma(E), \sqsubseteq^{R}, \sqsubseteq\right)$ is a distributive bicpo. We already know from Proposition 2 that $(\Gamma(E), \sqsubseteq)$ and $\left(\Gamma(E), \sqsubseteq^{R}\right)$ are cpos, and that $\sqsubseteq^{R}$-directed lubs are $\sqsubseteq$-lubs, which is the same as requiring that the identity function is continuous from $\left(\Gamma(E), \sqsubseteq^{R}\right)$ to $(\Gamma(E), \sqsubseteq)$. We also know that $(\Gamma(E), \sqsubseteq)$ is algebraic, which is easily seen to imply the continuity of $\sqcap$, provided binary glbs exist, which we prove now, using Axioms 4 and 5. Let $x$ and $y$ be two configurations. We define $x \sqcap y$ as the set of all events $e$ such that $e$ is in the $\leq^{L}$-downwards-closure of both $x$ and $y$, and is $\leq^{L}$-maximal with that property. This is clearly the glb of $x$ and $y$ if only it is a configuration. The definition ensures securedness by construction: if $e \in x \sqcap y$ and $e^{\prime} \leq^{R} e$, then $e^{\prime}$ is in the $\leq^{L}$-downwards-closure of both $x$ and $y$ by Lemma 3, and by definition of $x \sqcap y$ there exists $e^{\prime \prime}$ in $x \sqcap y$ such that $e^{\prime} \leq^{L} e^{\prime \prime}$. Suppose now that $e_{1}, e_{2}$ are in $x \sqcap y$. Let $e_{1}^{\prime}, e_{2}^{\prime}$ in $x$ be such that $e_{1} \leq^{L} e_{1}^{\prime}$ and $e_{2} \leq^{L} e_{2}^{\prime}$, and let $e_{1}^{\prime \prime}, e_{2}^{\prime \prime}$ in $y$ be such that $e_{1} \leq^{L} e_{1}^{\prime \prime}$ and $e_{2} \leq^{L} e_{2}^{\prime \prime}$. Since $e_{1}^{\prime}, e_{2}^{\prime} \in x$, we have $e_{1}^{\prime} \subsetneq e_{2}^{\prime}$, and similarly $e_{1}^{\prime \prime} \subsetneq e_{2}^{\prime \prime}$. We distinguish several cases.

- $e_{1}^{\prime} \neq e_{2}^{\prime}$. Here, by two applications of Axiom 4 we get first $e_{1} \frown e_{2}^{\prime}$ then $e_{1} \frown e_{2}$.

- $e_{1}^{\prime \prime} \neq e_{2}^{\prime \prime}$. We obtain $e_{1} \frown e_{2}$ similarly to the previous case.

- $e_{1}^{\prime}=e_{2}^{\prime}$ and $e_{1}^{\prime \prime}=e_{2}^{\prime \prime}$. By Axiom 5, $e_{1} \vee^{L} e_{2}$ exists. It is in the $\leq^{L_{-}}$ downwards-closure of both $x$ and $y$, which, by the definition of $x \sqcap y$, forces $e_{1}=e_{2}=e_{1} \vee^{L} e_{2}$.

In all cases, we have $e_{1} \subsetneq e_{2}$. This completes the proof of Axioms 1 and 2 .

We next check Axiom 3. Let $y \sqsubseteq^{R} z$ and $e \in x \sqcap y$. Then $e$ is in the $\leq^{L}$-downwards-closure of both $x$ and $z$. We show that $e$ is $\leq^{L}$-maximal with that property. Suppose that $e \leq^{L} e^{\prime}$ and $e^{\prime} \leq^{L} e_{1}^{\prime} \in x$ and $e^{\prime} \leq^{L} e_{2}^{\prime} \in z$. Now, for some $e_{3}^{\prime}$ in $y, e \leq^{L} e_{3}^{\prime}$ and thus, by Lemma 4 , we have that $e_{2}^{\prime}=e_{3}^{\prime} \in y$. Therefore, $e=e^{\prime}$ by the maximality property of $e$ with respect to $x$ and $y$. Hence $e \in x \sqcap z$, and we have proved $x \sqcap y \sqsubseteq^{R} x \sqcap z$.

To check Axiom 4, let subsets $S, S^{\prime}$ be $\sqsubseteq$-directed subsets of $\Gamma(E)$ such that whenever $s \in S$ and $s^{\prime} \in S^{\prime}$ there are $t$ in $S$ and $t^{\prime}$ in $S^{\prime}$ such that

$$
s \sqsubseteq t \text { and } s^{\prime} \sqsubseteq t^{\prime} \text { and } t \sqsubseteq^{R} t^{\prime} .
$$


By the proof of Proposition 2,

$$
\bigsqcup S=\left\{e \in \bigcup S \mid e \text { is } \leq^{L} \text {-maximal in } \bigcup S\right\}
$$

Equivalently, $\bigsqcup S$ consists of the events $e$ such that $e \in s$ for some $s$ in $S$ with the property that

$$
\forall t \in S(s \sqsubseteq t \Rightarrow e \in t) .
$$

Indeed, suppose that $e$ and $s$ satisfy property (1), and let $e_{1} \geq^{L} e$ be such that $e_{1} \in \bigcup S$, i.e., $e_{1} \in t$ for some $t$ in $S$. Let $s^{\prime}$ in $S$ be such that $s \sqsubseteq s^{\prime}$ and $t \sqsubseteq s^{\prime}$. Then $e \in s^{\prime}$ and $e_{1}$ is in the $\leq^{L}$-downwards-closure of $s^{\prime}$. This combined with $e \leq^{L} e_{1}$ yields $e=e_{1}$. Conversely, if $e \in s \in S$ and $e$ is $\leq^{L}$-maximal in $\bigcup S$, then $e, s$ obviously satisfy (1).

Given $e$ and $s$ satisfying (1), we wish to show $e \in \bigsqcup S^{\prime}$. By assumption, taking $s^{\prime}$ an arbitrary element of $S^{\prime}$, we have for some $t$ in $S$ and $t^{\prime}$ in $S^{\prime}$ that

$$
t \sqsubseteq^{R} t^{\prime} \text { and } s \sqsubseteq t \text { and } s^{\prime} \sqsubseteq t^{\prime} .
$$

By (1), we get that $e \in t^{\prime}$. Suppose $t^{\prime} \sqsubseteq t^{\prime \prime} \in S^{\prime}$. Replacing $s^{\prime}$ by $t^{\prime \prime}$, and reasoning as above, we get that $e \in t^{\prime \prime \prime}$ for some $t^{\prime \prime \prime}$ such that $t^{\prime \prime} \sqsubseteq t^{\prime \prime \prime}$. Finally, one easily derives that $e \in t^{\prime \prime}$ from $t^{\prime} \sqsubseteq t^{\prime \prime} \sqsubseteq t^{\prime \prime \prime}, e \in t^{\prime}$ and $e \in t^{\prime \prime \prime}$. Thus $e$ and $t^{\prime}$ satisfy property (1) relative to $S^{\prime}$, i.e., $e \in \bigsqcup S^{\prime}$. We conclude that $\bigsqcup S \sqsubseteq^{R} \bigsqcup S^{\prime}$, as required. Thus $\left(\Gamma(E), \sqsubseteq^{R}, \sqsubseteq\right)$ is a bicpo.

For distributivity, by Proposition 3 , we know that $\left(\Gamma(E), \sqsubseteq^{R}\right)$ is a distributive cpo. Now remark that if $x \uparrow^{R} y, x \sqsubseteq z$ and $y \sqsubseteq z$, then also $x \cup y \sqsubseteq z$ by the definition of $\sqsubseteq$.

Finally, to show that $\left(\Gamma(E), \sqsubseteq^{R}, \sqsubseteq\right)$ is a bidomain we need to produce an $\omega$-chain of finite projections on it whose least upper bound is the identity on $\Gamma(E)$. Here we refer to Proposition 6, showing how continuous stable functions on $\Gamma(E)$ correspond to configurations of the bistructure $! E \multimap E$; a configuration $F$ of $! E \multimap E$ corresponds to a continuous, stable function $\bar{F}: \Gamma(E) \rightarrow \Gamma(E)$ so that inclusion of configurations corresponds to the stable ordering of functions. Let $X$ be the configuration of $! E \multimap E$ corresponding to the identity function on $\Gamma(E)$. Because the events of $! E \multimap E$ form a countable set we may enumerate them as $e_{0}, e_{1}, \cdots, e_{n}, \cdots$. Define $X_{n}$ as the configuration

$$
\left[e_{0}\right]_{X} \cup \cdots \cup\left[e_{n}\right]_{X}
$$


Each $X_{n}$ is a finite set and so compact with respect to $\sqsubseteq^{R}$. By Proposition 6 , we obtain a chain of functions $\bar{X}_{n}, n$ in $\omega$, compact and increasing with respect to the stable order, with least upper bound the identity function on $\Gamma(E)$. Being stably less than the identity, each function $\bar{X}_{n}$ must be a projection.

We conclude that $\Gamma(E)$ is a bidomain.

Proposition 8 If $E_{0}$ and $E_{1}$ are B-bistructures, then so are $E_{0} \times E_{1}$ and $! E_{0} \multimap E_{1}$.

Proof. The verification for the product is immediate. We check Axioms 4 and 5 for $! E_{0} \multimap E_{1}$. Let $\left(x, e_{1}\right) \geq^{L}\left(y, e_{1}^{\prime}\right)$ and $\left(x, e_{1}\right) \frown\left(z, e_{1}^{\prime \prime}\right)$. Then $x \subseteq y, e_{1} \geq^{L} e_{1}^{\prime}$ and either $x \smile z$ or $e_{1} \frown e_{1}^{\prime \prime}$. If $x \smile z$ then $y \smile z$ (as $x \subseteq y$ ); if $e_{1} \frown e_{1}^{\prime \prime}$ then $e_{1}^{\prime} \frown e_{1}^{\prime \prime}$, by Axiom 4 for $E_{1}$. In either case it follows that $\left(y, e_{1}^{\prime}\right) \frown\left(z, e_{1}^{\prime \prime}\right)$. The verification of Axiom 5 is straightforward: $\left(x, e_{1}\right) \vee^{L}\left(y, e_{1}^{\prime}\right)=\left(x \Pi^{R} y, e_{1} \vee^{L} e_{1}^{\prime}\right)$, where we use Axiom 5 for $E_{1}$ and the fact that $\Gamma\left(E_{0}\right)$ is $\sqsubseteq^{R}$-bounded complete (cf. Proposition 2). [In boundedcomplete cpos, all non-empty glbs exist.]

Remark 5 On the other hand, Axiom 4 is not preserved by any of $\otimes,(\cdot)^{\perp}$ or !. Therefore we do not see how to get a model of linear logic with Bbistructures.

Theorem 2 The B-bistructures yield a full sub-cartesian closed category of the co-Kleisli category of the exponential comonad on BS. It is equivalent to a full sub-cartesian closed category of bidomains.

Proof. The first assertion is immediate from Proposition 8. By Proposition 7 and the last part of Theorem 1, the subcategory is equivalent to a full subcategory of the category of bidomains. It remains to show that there are isomorphisms of bidomains between $\Gamma\left(E_{0}\right) \times \Gamma\left(E_{1}\right)$ and $\Gamma\left(E_{0} \times E_{1}\right)$, and also between $\Gamma\left(E_{0}\right) \rightarrow \Gamma\left(E_{1}\right)$ and $\Gamma\left(! E_{0} \multimap E_{1}\right)$. The first is easy to see (it is $(x, y) \mapsto x \cup y$, assuming $E_{0}$ and $E_{1}$ disjoint). The second is given by $f \mapsto \operatorname{tr}(f)$, by virtue of the remarks above on function spaces of bidomains and Proposition 6.

Notice that if $\leq^{L}=i d$, Axioms 4 and 5 hold vacuously, hence the category of B-bistructures is large enough to contain the models of typed $\lambda$-calculi where the base types are interpreted by event structures. 


\section{Discussion}

In this section, we discuss possible variations and connections with other work.

Variations. Besides coherence spaces there are a few other "web-based" models of intuitionistic linear logic or full classical linear logic. Most notably, there are Ehrhard's hypercoherences with a Kleisli category of strongly stable functions, which can be seen as an extensional (although not orderextensional) account of sequentiality. At first order, the strongly stable model contains exactly the sequential functions. At higher orders, it is the extensional collapse of the model of sequential algorithms [8,9]. Generalisations encompassing both Girard's webs and hypercoherences have been proposed independently by Lamarche [18] (based on quantale-valued sets) and by Winskel [30] (based on a notion of indexing inspired by logical relations). We believe that our biordering treatment can be applied to all these categories. We checked this for hypercoherences. There, the binary coherence relation $\smile$ is replaced by a coherence hypergraph $\Gamma$. Thus, a hypercoherence is given by a set $E$ of events and a set $\Gamma$ of finite non-empty subsets of $E$. We axiomatise "hypercoherence-bistructures" exactly like bistructures, replacing $\frown$ by $\Gamma$, and Axiom 3 by:

$3(a) \quad\left(X \in \Gamma\right.$ and $X$ is $\leq^{L}$-lower bounded $) \Rightarrow X$ is a singleton,

$3(b) \quad X$ is $\leq^{R}$-upper bounded $\Rightarrow X \in \Gamma$

The variation works smoothly because there is very little interaction between the axiomatisation of the coherence structure and that of the orders. The two propagate smoothly in the construction of the connectives, and are related only through Axiom 3 or its variants.

Although the bidomain model incorporates both the stable and pointwise order, its PCF theory (those inequalities on terms which hold in the bidomain model) does not include that of the Scott model. The argument follows Jim and Meyer's in [13], and is based on the bidomain model failing to eliminate an "or-tester", the first of Curien's examples in [5], Proposition 4.4.2. The same argument applies to the variation sketched above, where coherence is replaced by hypercoherence. In [29] it is shown how to adjoin an additional conflict relation to bistructures to obtain a model of PCF whose 
theory strictly includes that of the Scott model. Several strengthenings of this idea are possible; the conflict relation can be combined with hypercoherences on bistructures, or even replaced by coherence relations of a more complex kind (akin to logical relations, as in [30]).

As remarked in the introduction, by Loader's result [19] there is no hope of finding a direct presentation of the fully abstract model for PCF in this way, adjoining a suitable coherence structure to the two orders of bistructures, unless, as in [22], this is of an infinitary nature. However, the fact that there are hypercoherence-based bistructure models refining the Scott model shows (adapting the "Definability Lemma", e.g., Proposition 4.1.10 of [5], to a slightly broader setting) that there are extensions of PCF, having some claims to being sequential and functional, but which nevertheless have an effectivelygiven fully abstract model. Such extensions to PCF escape Loader's proof. But the precise programming constructs associated with these extensions is a mystery, and would need a deeper understanding of the operational nature of bistructure models.

Connections. Lamarche [17] (followed by Kegelmann [15]) has proposed a large cartesian closed category of "interpolative domains" encompassing, as full sub-cartesian closed categories, categories of continuous functions on one hand, and categories of stable functions on the other. Lamarche's framework has some technical similarities with ours; two orders are axiomatised, with a factorisation property. But the approach and goals are quite different. In Lamarche's setting, once the standard interpretation of the basic flat domains of, say, PCF, is fixed appropriately, the larger category induces exactly the continuous or stable model, whereas we obtain a model simultaneously refining both the stable and continuous models.

\section{Acknowledgements}

Gordon Plotkin's research was supported by an EPSRC Senior Fellowship. 


\section{References}

[1] Abramsky, S., Jagadeesan, R., and Malacaria, P., Games and full abstraction for PCF, manuscript available from http://www.dcs.ed.ac.uk, 1995.

[2] Berry, G., Modèles complètement adéquats et stables des lambda-calculs typés. Thèse de Doctorat d'Etat, Université de Paris VII, 1979.

[3] Berry, G., Curien, P.-L. and Lévy, J.-J., The full abstraction problem: state of the art, in Proc. French-US Seminar on the Applications of Algebra to Language Definition and Compilation, Cambridge University Press, 1985.

[4] Bierman, G.M., What is a categorical model of intuitionistic linear logic?, in Proc. 2nd. Int. Conf. on Typed Lambda Calculi and Applications (eds. Dezani-Ciancaglini, M. and Plotkin, G.), LNCS, Vol. 902, pp. 78-93, Springer-Verlag, 1995.

[5] Curien, P.-L., Categorical combinators, sequential algorithms, and functional programming. Second edition, Birkhäuser, 1993.

[6] Curien, P.-L., On the symmetry of sequentiality, in Proc. 9th Int. Conf. on Mathematical Foundations of Programming Semantics (eds. Brookes, S., Main, M., Melton, A., Mislove, M. and Schmidt, D.), LNCS, Vol. 802, pp. 29-71, Springer-Verlag, 1994.

[7] Curien, P.-L., Coherence bistructures, unpublished manuscript, Beijing, 1994.

[8] Ehrhard, T., Hypercoherences: a strongly stable model of linear logic. MSCS, Vol. 3, No. 4, pp. 365-385, 1993.

[9] Ehrhard, T., A relative PCF-definability result for strongly stable functions, available by ftp at iml.univ-mrs.fr, in directory /pub/ehrhard/rel.[dvi,ps].Z, 1996.

[10] Girard, J-Y., Linear logic. TCS Vol. 50, No. 1, pp. 1-102, 1987.

[11] Girard, J-Y., Lafont, Y., and Taylor, P., Proofs and types. Cambridge University Press, 1989. 
[12] Hyland, J.M.E., and Ong, C-H.L., On full abstraction for PCF: $I$, II and III, available by ftp at ftp.comlab.ox.ac.uk, in directory /pub/Documents/techpapers/Luke.Ong/pcf.ps.gz, 1994.

[13] Jim, T., and Meyer, A., Full abstraction and the context lemma. in Proc. 1st. Int. Conf. on Theoretical Aspects of Computer Software (eds. Ito, T. and Meyer, A.R.), Sendai, LNCS Vol. 526, pp. 131-152, Springer-Verlag, 1991.

[14] Kahn, G. and Plotkin, G., Concrete Domains, in A Collection of Contributions in Honour of Corrado Böhm on the Occasion of his 70th Birthday. TCS Vol. 121, Nos. 1-2, pp. 187-278, 1993.

[15] Kegelmann, M., Factorization systems on domains, Technical Report, School of Computer Science, The University of Birmingham, 1996.

[16] Lamarche, F., Sequentiality, games and linear logic, in Proc. CLICS Workshop, Aarhus University, Aarhus University DAIMI PB-397 (II), 1992.

[17] Lamarche, F., A large cartesian closed category of domains, to appear in Inform. and Comp.

[18] Lamarche, F., Generalizing coherence spaces and hypercoherences, in Proc. 11th. Int. Conf. on Mathematical Foundations of Programming Semantics (eds. Brookes, S., Main, M., Melton, A. and Mislove, M.), Electronic Notes in Theoretical Computer Science, pp. 259-268, NorthHolland, 1995.

[19] Loader, R., Finitary PCF is not decidable. Submitted for publication, 1996.

[20] Milner, R., Fully Abstract Models of Typed $\lambda$-calculi, in Theoretical Comp. Sci., Vol. 4, pp. 1-22, 1977.

[21] Nielsen, M., Plotkin, G., and Winskel, G., Petri nets, event structures and domains, part I. TCS, Vol. 13, No. 1, pp. 85-108, 1981.

[22] O'Hearn, P.W. and Riecke, J.G., Kripke logical relations and PCF. Inform. and Comp., Vol. 120, No. 1, pp. 107-116, 1995. 
[23] Plotkin, G., and Winskel, G., Bistructures, bidomains and linear logic, in Proc. 21st ICALP (eds. Abiteboul, S., and Shamir, E.), LNCS, Vol. 820, pp. 352-363, Springer-Verlag, 1994.

[24] Seely, R.A.G., Linear logic, $\star$-autonomous categories and cofree coalgebras, in Categories in Computer Science and Logic (eds. Gray, J.W. and Scedrov, A.), Contempory Mathematics, Vol. 92, pp. 371-382, American Mathematical Society, 1989.

[25] Winskel, G., Events in computation. Ph.D. thesis, University of Edinburgh, CST-10-80, 1980.

[26] Winskel, G., Event structures, in Petri Nets: Applications and Relationships to Other Models of Concurrency, Advances in Petri Nets 1986, Part II; Proceedings of an Advanced Course, (eds. Brauer, W., Reisig, W. and Rozenberg, G.), LNCS, Vol. 255, pp. 325-392, SpringerVerlag, 1987.

[27] Winskel, G., An introduction to event structures, in REX School/Workshop on Linear Time, Branching Time and Partial Order in Logics and Models for Concurrency, (eds. de Bakker, J.W., de Roever, W.P. and Rozenberg, G.), LNCS, Vol. 354, pp. 364-397, Springer-Verlag, 1989.

[28] Winskel, G., The formal semantics of programming languages, MIT Press, 1993

[29] Winskel, G., Stable bistructure models of PCF, in Proc. 19th Int. Conf. on Mathematical Foundations of Programming Semantics, (eds. Prívara, I., Rovan, B., and Ružička, P.,) LNCS, Vol. 841, SpringerVerlag, 1994.

[30] Winskel, G., Hypercoherences, handwritten notes, 1994.

[31] Zhang, G.Q., Logic of domains. Birkhäuser, 1991. 